\title{
COLLEGIATE E MONASTERI NEL BASSO ADIGE TRA LA SECONDA METÀ DEL X E LA FINE DELL'XI SECOLO
}

\section{GIAN PIETRO BROGIOLO}

UDC: $726.71(450.32) " 09 / 10 "$

Original scientific paper

Manuscript received: 28. 02. 2017.

Revised manuscript accepted: 13. 03. 2017.

DOI: 10.1484/J.HAM.5.113719
G. P. Brogiolo

Via dei Ronchi 28

25080-Polpenazze del Garda

Italia

gpbrogiolo@gmail.com

Il territorio del basso Adige, già dipendente dalla città romana di Este e in età carolingia comitato di Monselice, tra il 952 (istituzione della Marca di Verona da parte di Ottone I) e la fine dell'XI secolo (nell'età di Matilde di Canosssa) diviene strategico nei rapporti tra l'impero e l'emergente Venezia. Vi dominano personaggi di grande rilievo pubblico che, in qualità di marchesi, si muovono al vertice dell'impero: da Almerico II, morto nel 955, a Ugo il grande di Toscana (dal 970 al 1001) ad Alberto Azzo I (agli inizi dell'XI secolo). Il fglio di questi, Alberto Azzo II (996-1097), è il padre del duca di Baviera Guelfo IV ed uno dei protagonisti della lotta per le investiture al fianco di Matilde che nel 1089, a 43 anni, ne sposa il sedicenne nipote Guelfo V. Il ruolo di primo piano di queste aristocrazie si coglie, nel basso Adige, nell'alto livello architettonico delle fondazioni religiose, delle quali, grazie a recenti ricerche, è ora possibile proporre piante, sequenze e ipotesi di datazione. In questo contributo vengono analizzati due complessi (Santa Maria della Vangadizza e Santa Maria di Carceri), fondati come collegiate poco prima, rispettivamente, del 955 e del 1078, trasformati poi entrambi in monasteri.

Keywords: Matilde of Canossa, Alberto Azzo II, abbey of Vangadizza, abbey of Carceri, saint Theobald, romanesque achitecture

\section{IL CONTESTO STORICO DA ADELAIDE DI BORGOGNA (CA. 931- 999) A MATILDE DI CANOSSA (1046-1115)}

Due grandi donne, Adelaide di Borgogna (ca. 931-999) e Matilde di Canossa (1046-1115), entrambe elevate all'onore degli altari per essersi schierate dalla parte della Chiesa, sono tra i protagonisti delle vicende dinastiche attorno alla qualti ruotano le fondazioni religiose trattate in questo contributo.

La vita di Adelaide, nata ca. 931, è quella di un'eroina romantica: dopo la morte del marito Lotario, figlio del re d'Italia Ugo di Provenza, nel 951 viene imprigionata nella Rocca di Garda per ordine dell'imperatore Berengario II. Riuscita a fuggire, sposa Ottone I che sconfigge Berengario e diventa imperatore. Dopo la sua morte, terrà la reggenza per il figlio Ottone II. Morto precocemente anche lui nel 983, continuerà ad avere un ruolo di primo piano accanto alla nuora Teofano, reggente per il figlio Ottone III. Adelaide è dunque al vertice del potere nei cinquant'anni nei quali la storia d'Europa cambia grazie alla Renovatio Imperii attuata dagli Ottoni.

In questo nuovo quadro politico, un grande sviluppo ha anche il Nord Est italiano a seguito dell'istituzione, nel 952, della Marca di Verona, strettamente collegata ai ducati di Carinzia e di Baviera. Nella Marca il fiume Adige assume un ruolo chiave. Nel primo tratto di montagna (dal Trentino a Verona), nella valle dell'Adige si snoda il principale percorso tra le due regioni più importanti dell'Impero (i regni di Germania e d'Italia) e Roma, sede del papa dal quale dipende la concessione della corona imperiale. A sud di Verona, dove il potere dal IX secolo è affidato alla famiglia degli Almerici, il fiume porta al mare e a Venezia (fig. 1), città chiave per $\mathrm{i}$ rapporti tra il Sacro Romano Impero e l'Impero bizantino. Rapporti rinsaldati, al vertice, dal matrimonio di Ottone II con la principessa Teofano e, ad un gradino inferiore, dal matrimonio (dopo il 959 e prima del 963) tra il doge Pietro IV Candiano e Waldrada, sorella di Ugo il grande'. Personaggio di grande rilievo per lignaggio (era figlio del marchese Uberto e nipote del re Ugo di Provenza e suo zio Lotario aveva sposato, come ho detto, Adelaide), per i rapporti diretti con i tre imperatori Ottone I, II e III, è marchese di Toscana per oltre un trentennio (prima dell'aprile del 970-1001) e duca di Spoleto per dieci anni (dal 986 al 996)². Ad Ugo erano anche passati i beni di Almerico II (955), fondatore della collegiata di Santa Maria della Vangadizza e discendente della famiglia che dal IX secolo deteneva il potere nel basso Adige per conto dell'imperatore.

Dopo Ugo, pure lui morto senza eredi nel 10o1, il controllo del basso Adige e la collegiata passano agli Obertenghi, il più importante dei quali è Alberto Azzo II, morto a 101 anni nel 1097: marchese di Lunigiana e conte di Milano, aveva sposato, tra 1034 e 1036, Cunizza, figlia di Guelfo II, conte di Aldorf e duca di Carinzia e di Imiza, e parente dei duchi di Baviera e dell'imperatore Enrico II. Più di Ugo, i cui interessi si mossero prevalentemente tra la Toscana e la Germania (ad Ingelheim, nel 994, aveva costruito una dimora vicina al palazzo dell'imperatore 3 ), Alberto Azzo II, fin dagli anni ' 30 dell'XI secolo attivo nel distretto di Monselice, nella seconda

\footnotetext{
${ }^{1}$ Pietro IV, dapprima filoberengariano, si era poi avvicinato ad Ottone I e Ottone II e aveva sposato, prima del 963, Waldrada dopo aver ripudiato, e relegato come badessa del monastero ducale di San Zaccaria, la prima moglie Giovanna, discendente di una famiglia aristocratica di Ravenna. Forse a causa di contrasti familiari (lui stesso si era ribellato al padre che lo aveva associato al ducato ed era stato costretto a fuggire da Venezia), nel 976, nel corso di una rivolta, venne ucciso assieme al bambino avuto da Waldrada. Lo stesso anno, in un placito tenuto a Piacenza, alla presenza dell'imperatrice Adelaide, Waldrada ratifica e rende pubblico il documento con cui dichiara di aver riavuto dal nuovo doge, Pietro I Orseolo, il suo morgengab (C. PROVESI, Le due mogli di Pietro IV Candiano (959 976): le donne e i loro gruppi parentali nella Venezia del X secolo, "Reti Medievali Rivista", 16, 2 (2015), <http://www.retimedievali.it>, p. 35). ${ }^{2}$ A. PUGLIA, Vecchi e nuovi interrogativi sul marchese Ugo di Tuscia (970-1001), in Dalle abbazie, l'Europa. I nuovi germogli del seme benedettino nel passaggio tra primo e secondo millennio (secc. X-XII), in A. Guidotti, G. Cirri 2006, I nuovi germogli del seme benedettino nel passaggio tra primo e secondo millennio (secc. X-XII), (Badia a Settimo, 22-23 maggio 1999), Firenze 2006, pp. 151-186.
}

${ }^{3}$ A. PUGLIA, Vecchi e nuovi interrogativi... 


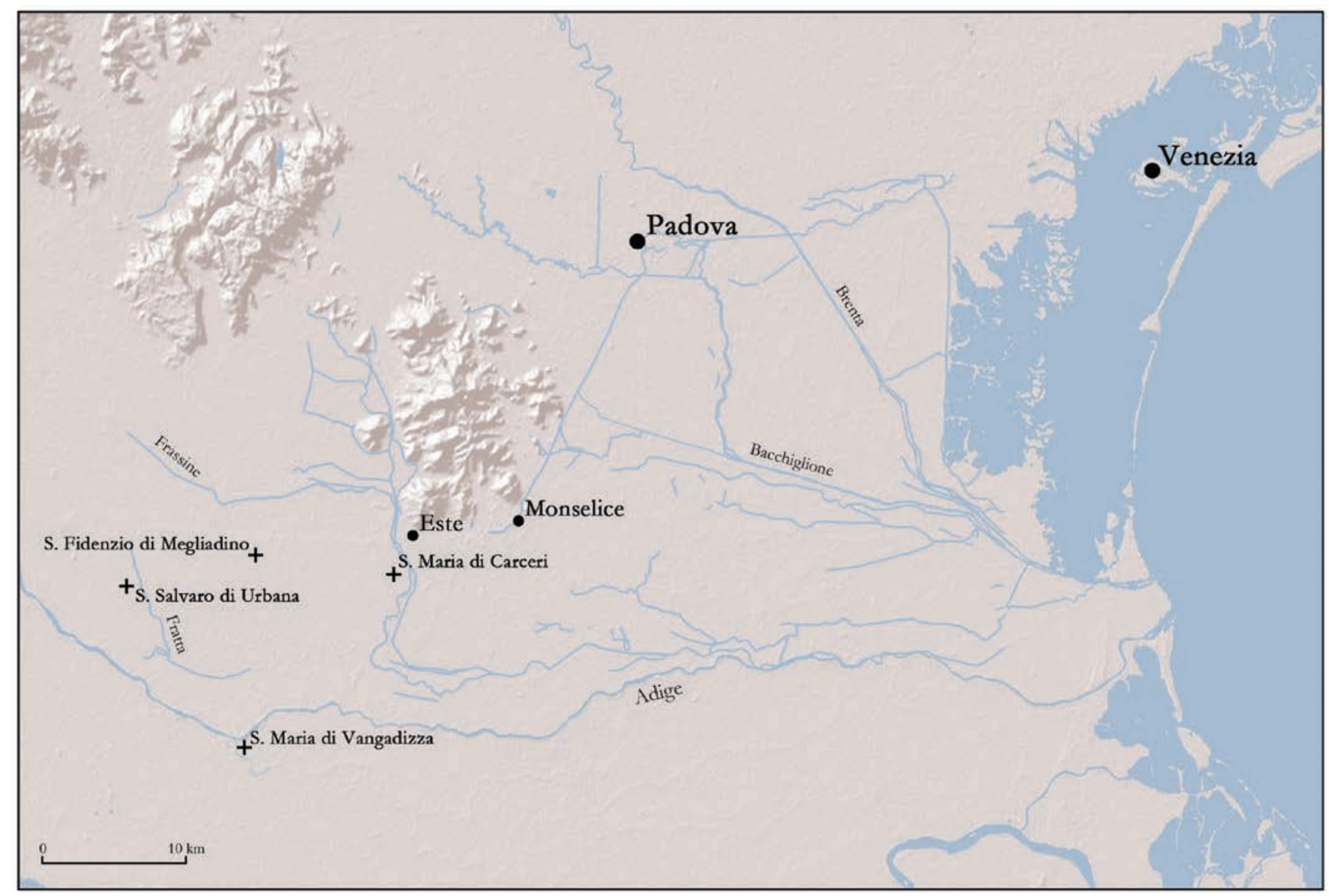

Fig. 1. Il fume Adige con Este e le chiese di Megliadino, Urbana, Vangadizza e Carceri.

metà del secolo fissa la sua residenza nel castello di Este, dando vita alla casata degli Estensi che da quel castello deriverà il nome. Particolarmente stretti furono i suoi rapporti con Matilde di Canossa che nel 1089 (a 43 anni) ne sposa il nipote sedicenne Guelfo V. Marchesa di Toscana e contessa di Ferrara, Matilde è la celebre protagonista di un periodo tra i più importanti della storia europea, segnato come fu dalla lotta per le investiture, che vide contrapposti il papa Gregorio VII e l'imperatore Enrico IV. Accanto a loro, in prima linea, ebbero un ruolo di primo piano anche i vescovi di Padova. Dapprima Olderico (1064-108o) che da legato del papa Gregorio VII passa poi dalla parte dell'imperatore e infine viene assassinato per mano di un uomo della scorta, mentre, di ritorno dalla Germania, si recava a Roma. Una ventina d'anni dopo, il filopapale Sinibaldo, deposto dall'imperatore, si rifugia ad Este sotto la protezione dei marchesi e potrà tornare a Padova solo dopo il concordato $\mathrm{d}$ Worms (1122) che pone fine alla lotta per le investiture4.

$\mathrm{E}$ in questo contesto storico, e in relazione alle famiglie che detennero in potere nel basso Adige tra X e XI secolo, che si collocano le vicende, oltre che di Santa Maria della Vangadizza (ante 955), anche della più recente (ca. 1078) Santa Maria di Carceri. Di questi due complessi, incrociando fonti scritte e dati materiali, è possibile ricostruire la storia, inquadrandola nello sviluppo economico e nella cultura architettonica che si sviluppò, al più alto livello, nelle relazioni tra il regno italico e Venezia

\section{SANTA MARIA DELLA VANGADIZZA, COLLEGIATA, MONASTERO E MAUSOLEO}

Nonostante Santa Maria della Vangadizza sia stato uno dei monasteri più importanti del Veneto, non è stato sinora oggetto di uno studio sistematico. L'archivio, spezzettato al momento della soppressione, è completamente inedito, salvo pochi documenti pubblicati nel '70o negli Annales Camaldulenses. La chiesa è ridotta allo stato di rudere (fig. 2) e dello scavo del suo interno, eseguito nel 2003 e reinterrato, non è stata pubblicata alcuna informazione, salvo una pianta cumulativa e un paio di foto reperibili in internet ${ }^{5}$. Quanto sappiamo dalle fonti scritte, integrato da quel che si può ancora osservare in in alzato, è peraltro sufficiente per delinearne una storia plausibile.

\subsection{La chiesa nelle fonti scritte}

Almerico II e la moglie Franca, prima del 955, avevano fondato una chiesa dedicata a Santa Maria Vergine alla Van-

\footnotetext{
${ }_{4}^{4}$ P.F. ZATTA, Storia dei vescovi di Padova, Roma 2015.

${ }^{5}$ La chiesa di Santa Maria è stata oggetto nel 2003 di uno scavo, ancora inedito, diretto da F. Zuliani e A. Nicoletti. I dati, conservati nell'archivio della Soprintendenza, non solo disponibili e le strutture sono state interamente ricoperte. In rete si trovano però una pianta e alcune foto degli scavi http://www. archeoed.it/index.asp?lang=it\&col=1\&section=progetti\&IDprogetto=22. Le immagini sono state scaricate il 25 ottobre 2015. Una rapida lettura stratigrafica e un nuovo rilievo planimetrico e prospettico sono stati eseguiti il 20 ottobre 2015 grazie alla disponibilità del Comune di Badia Polesine, proprietario del complesso monastico. Le pagine che seguono non vogliono essere un'invasione di campo nella ricerca di due ex colleghi, ma solo un incentivo affinché pubblichino, se non un testo definitivo, quantomeno una notizia preliminare su questo importante monumento, senza il quale non si può apprezzare il contributo di Alberto Azzo II e della sua famiglia all'architettura dell’XI secolo.
} 
gadizza (Badia Polesine, in provincia di Rovigo), in loco et fundo qui vocatur flumen Vedre e l'avevano affidata a Johannes presbiter custos et rector. La donazione per la collegiata (schola sacerdotum), istituita presso la chiesa, comprendeva beni a Cavazzana, a nord est di Lendinara, e alcuni diritti pubblici di natura fiscale, evidentemente detenuti dal marchese, relativi ai transita ... in alveo fluvio Athesis qui vocatur Flumen Vedre prope iam dicta ecclesia sancte Marie ${ }^{6}$.

Alcuni mesi più tardi, il 6 dicembre, Franca, stando nel castello di Rovigo, con il consenso di Indemario presbiter et mundualdus (rimasta vedova, secondo la legge longobarda non poteva agire da sola in un atto pubblico), dona per l'anima sua e del marito, altri cospicui beni alla basilica di Santa Maria, da loro noviter fundata 7 . In aggiunta, rispetto

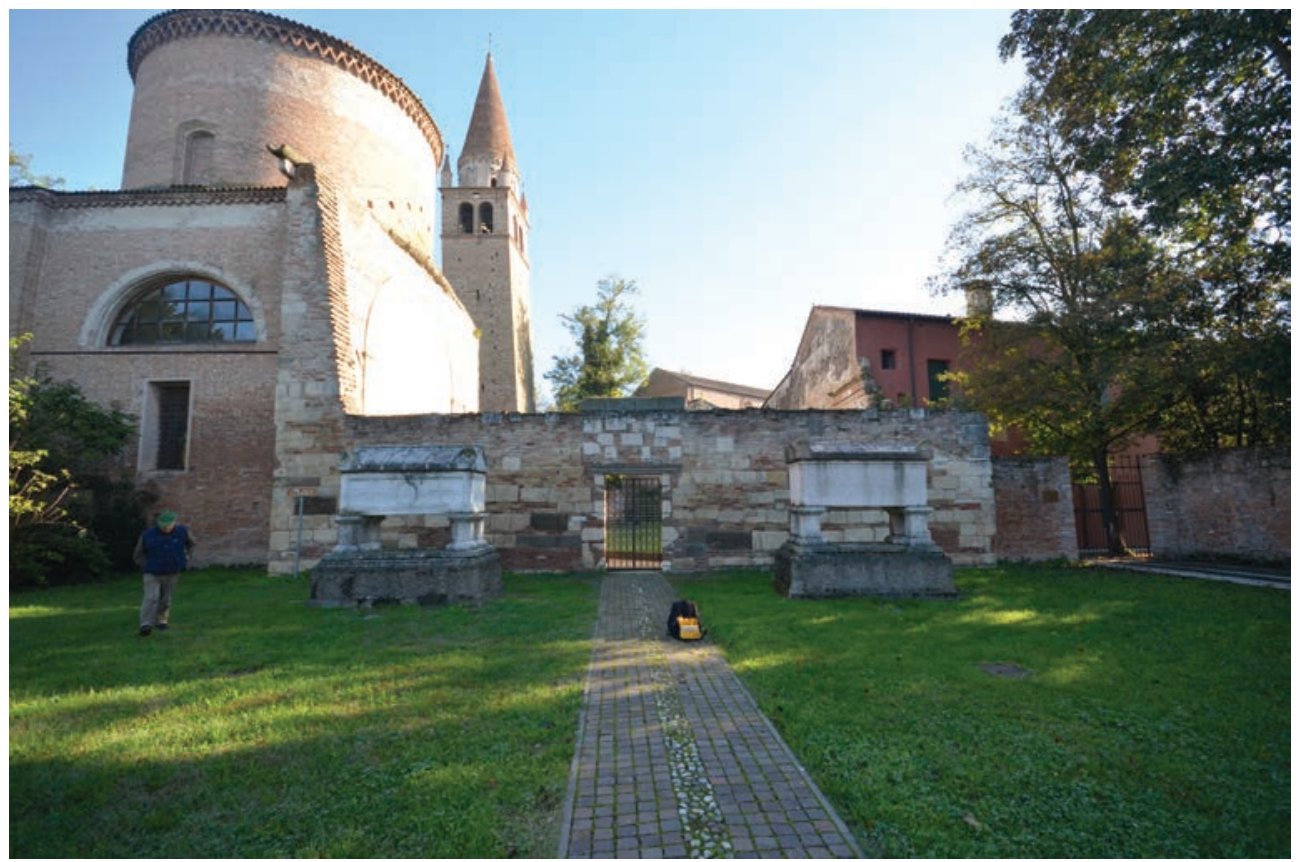
al documento di agosto, si specifica che la chiesa si trova in castro de Adice maiore ... iuxta Fluvio Adexe veglo, castello costruito su proprietà (in propria re) da Almerico II ${ }^{8}$. La scelta dei beni, donati da Franca, assicura alla chiesa una rendita equilibrata tra le aziende agricole e i molti spazi incolti o in fase di conquista agraria, testimoniati, nel territorio di Badia, dalla toponomastica medievale (Boscovecchio, Gazzo, Guasto Brusà, Ceresolo, Carletti, Colomban, Schiesara).

Prima del 30 maggio del 961 la collegiata e i beni di Almerico II sono finiti, come si è già detto, nelle disponibilità del marchese Ugo il grande di Toscana9 ${ }^{9}$. In quella data, infatti,

Fig. 2. Vangadizza, Santa Maria.

i re Berengario II ed Adalberto, a seguito della petizione del marchese ${ }^{10}$, donano a Martino, wangadiciensis monasterii abatem (definizione che fa ipotizzare la trasformazione della collegiata in cenobio), una "terra" presso l'isola di Carpi, dipendente dalla curia Leniaci e confinante con una selva fino al fiume Tartaro, già proprietà del monastero" ${ }^{11}$. Trent'anni più tardi, il 27 aprile del 993, il medesimo Ugo, pro remedio dell'anima sua, del padre Uberto, della madre Willa e di tutti i parenti, dona la chiesa, con tutte le proprietà concesse dal marchese Almerico II, all'abate Martino, affinchévi istituisca un monastero secondo la regola di san Benedetto ${ }^{12}$. Lo stes-

\footnotetext{
${ }^{6}$ Codice diplomatico padovano dal secolo VI a tutto l'undecimo, a cura di A. Gloria, Venezia 1877 (d'ora in avanti CDP, I), n. 43, con data agosto 955. ${ }^{7}$ La chiesa è la stessa della donazione precedente almeno per tre motivi: (a) custos et rector è sempre il presbiter Johannes; (b) viene ricordata la precedente concessione dei diritti di transito sul fiume (tolloneum de navibus fluminis Adixe veglo quam ad tolloneum ad sancta Maria translatum fuit); (c) la chiesa è effettivamente una collegiata presso la quale risiedono, o risiederanno in futuro, sacerdotes, levites seu alii clerici, uno dei quali (il presbitero Ildemario) assiste all'atto. S. COLLODO (Le chiese del marchese Almerico II e della moglie Franca (955), "Terra e storia", II, 2013, n. 4, pp. 13-67) ritiene invece che la donazione di Franca si riferisca alla canonica di Santa Maria delle Carceri (pp. 43-49), menzionata per la prima volta nel 1078 (M. VIGATO, Il monastero di S. Maria delle Carceri, i comuni di Gazzo e Vighizzolo, la comunità atestina. Trasformazioni ambientali e dinamiche socio-economiche in un'area del basso padovano tra medioevo ed età moderna, Carceri (PD) 1997, p. 19). La proposta è però difficile da sostenere, in quanto: (a) pur se a Carceri vi è traccia di un vecchio percorso dell'Adige, questo non è certo medievale; (b) attorno alla chiesa di Carceri non vi è alcuna evidenza di un castello; (c) tutte le case massariciae, oggetto della donazione del 955, si trovano più ad ovest, nella Scodosia, a sud di Montagnana e non lontano da Badia.

${ }^{8} \mathrm{CDP}$, I, n. 44. Oltre al teloneo quod dicitur trexidura de navibus, imposta diretta applicata sulle merci pagata nei porti di transito e scalo, Franca aggiunge: (a) sei case massaricie a Merlara, Altaura (con due vigneti, uno dei quali detto Grimialdola), Casale di Scodosia (infra villa Casale que cernit de uno latere terra deserta Sculdaxia et da uno latere fossato et terra et piscaria de Est qui a fundo... et da alio capite terra vigra seo omnibus que fuerunt iuris mei de Gondo), Urbana, Perariolo e i censi dei diritti di pesca e di caccia tra la rupta de Adice et de Visinaria decorente remissa de Tartaro per Stafolo (termine longobardo che indica probabilmente un cippo di confine) decorente in suprascrita rupta de Cadinario; (b) la peschiera di Corliano; (c) la vicina selva que vocatur Gurgo de Muro, que cernit fines de uno latere Liverno percorrente usque in fossa da Molino.

9 Nella donazione del 993 si specifica che la chiesa fondata dal marchese Almerico II in capite de Flumine Veclo è di sua proprietà e viene donata con tutti i beni da essa dipendenti.

${ }^{10}$ Identificato con il figlio del marchese Oberto che, ancora bambino, sarebbe stato associato al padre, grazie ad un patto con i re d'Italia (G. VEDOVATO, Ugo di Tuscia..., p. 188. Per A. PALLAVICINO (Le parentele del marchese Almerico II, in A. Spicciani, M. Tangheroni, Formazione e strutture dei ceti dominanti nel medioevo: marchesi, conti e visconti nel Regno Italico (secc. IX-XII), (Pisa, 18-20 marzo 1999), Roma, Istituto storico italiano per il medio evo (Nuovi studi storici 56), Roma 2003, pp. 233-320, a p. 244) sarebbe figlio del conte di Modena Suppone il Nero.

"Con tutte le relative pertinenze: cum terris, pratis, sulvis, paludibus, saletis, sacionibus, stalareis, ripis, rupinis, molendinis, piscationibus, pascuis, mercatis,, vallibus, planeciis, aquis aquarumque decursibus (Annales Camaldulenses ordinis sancti Benedicti tomus primus complectens res gestas ab anno Christi 907. ad annum 1026, a cura di G.B. Mittarelli, A. Costadoni, Venezia, 1755 (da qui in avanti Annales Camaldulenses), I, n. 25; Codice diplomatico veronese, a cura di V. Fainelli, I (Venezia 1949), II (Venezia 1963), da qui in avanti CDV, I, n. 206; E. ROSSINI, L'Adige e l'abbazia della Vangadizza, "Atti e Memorie del Sodalizio Vangadiciense", I, 1972-1973 p. 201-225, a p. 208).

${ }^{12}$ Annales Camaldulenses, I, n. 53. Considero inattendibili due ipotesi, recentemente formulate: la prima propone una distinzione tra la collegiata fondata da Almerico II e Franca (ubicata dove si insediò il monastero) e un monastero fondato nel sobborgo tra Francavilla e Salvaterra (C. CORRAIN, E. ZERBINATI, Vangadizza e Petra, "Wangadicia", 6-8, 2010, pp. 13-26); la seconda ipotizza che i documenti si riferiscano a tre differenti siti, tra Santa Maria di Carceri, il monastero della Vangadizza di Legnago, e quello omonimo di Badia (S. COLLODO, Le chiese del marchese Almerico II...).
} 
so Ugo ottiene poi dal papa Silvestro II (999-1003), con cui aveva rapporti di amicizia, un privilegium libertatis, ovvero la protezione apostolica del monastero ${ }^{13}$. Tre successivi atti, rogati tra il 996 e il 997, riguardano ulteriori donazioni, ad opera di Ugo il grande di Toscana e con il coinvolgimento della sorella Waldrada ${ }^{14}$.

Morto Ugo il grande nel 10o1, le sue proprietà passano agli Obertenghi che fanno della Vangadizza il principale punto di riferimento religioso della famiglia. Nel maggio del 1013 Alberto Azzo I ed il fratello Ugo, entrambi marchesi, presiedono un placito a Monselice alla presenza del conte padovano Todello, non sappiamo se perché investiti di una qualche autorità pubblica nella giudicaria di Monselice o come missi dominici dell'imperatore. La questione riguarda una controversia che contrappone proprio il monastero della Vangadizza, del quale avevano probabilmente assunto il patrocinio, al potente cenobio veneziano di San Zaccaria, fondato dal doge. Nel 1040 il normanno Rodolfo elargisce una donazione alla chiesa pro memoria del marchese Ugo, plausibilmente sepolto presso il monastero. Due nuove importanti donazioni si hanno nel 1073, da parte di Guelfo IV, figlio di Alberto Azzo II e duca di Baviera dal 1070, poi di Alberto Azzo II (nel 1075). In questa si ricorda la presenza nella chiesa del corpo di San Teobaldo (proclamato santo nel 1073) e della tomba di Cunizza, moglie di Alberto Azzo II, morta vent'anni prima.

\section{LA COLLOCAZIONE NELLA CHIESA DEL CORPO DI SAN TEOBALDO}

In un'epigrafe, apposta nella parete meridionale della chiesa (su un paramento che non è quello originale), l'abate Orlando ricorda il rinvenimento, nel 1226, dei corpi dei santi Primo, Feliciano ${ }^{15}$ e Teobaldo (ANNO DOMINI/ MCCXXVI HOC IN/ TEMPLO NON EST OPUS PULC/RIUS ISTO.
QUANDO FUIT/ FACTUM CORPORA/ SANCTORUM PRIMI ET FELICIANI/ ET SANCTI THEOBALDI IN/VENTA FUERUNT. TU QUI/CUMQUE LEGIS, SCIAS QUOD ABB/ AS ORLANDUS HOC OPUS FIERI FECIT) ${ }^{16}$. Gli annalisti precisano che il ritrovamento si verificò in occasione di un restauro parziale ${ }^{17}$.

Mentre le reliquie dei santi Primo e Feliciano erano probabilmente conservate nella chiesa fin dalla fondazione, il corpo di san Teobaldo vi era stato trasferito prima del 22 settembre del 1075, data nella quale Alberto Azzo II elargisce doni al monastero di Santa Maria e San Teobaldo ubi sanctorum corpora Primi et Feliciani requiescunt, nec non et corpus beatissimi quoque confessoris Teobaldi adesse videtur ${ }^{18}$. s. Teobaldo era figlio di Arnolfo, conte dello Champagne e, come racconta la Vita, scritta da Pietro abate della Vangadizza ${ }^{19}$, morì nel 1066 nell'eremo di Sajanega nei pressi di Sossano (Vicenza). Sette anni dopo la morte, nel 1073, era già stato proclamato santo, probabilmente grazie all'intervento di Alberto Azzo II sul papa. Ed è significativo il parallelo con la strategia adottata a Padova, alla fine del 1075, dal vescovo Olderico, che promuove, d'accordo con l'abate di Santa Giustina, il ritrovamento del corpo di s. Daniele e il suo trasferimento nella cattedrale da lui ricostruita.

Dopo la inventio del 1226, il corpo di s. Teobaldo era stato deposto in una cassa lignea davanti all'altare a lui dedicato, cassa che venne riaperta il 23 maggio del 1411 e sostituita, due giorni dopo, con una nuova ${ }^{20}$. In una ulteriore ricognizione, effettuata il 31 marzo $1617^{21}$, venne aperta la "cassa di marmo nella quale trovarono un'altra cassa di legno col coperto pur di legno, e sopra le reliquie un panno di lino sottile vergato di seta verde, sopra il quale era una croce di legno grossamente fabbricata; sotto questa trovarono tutte l'ossa d'un uomo poste secondo l'ordine naturale di un corpo umano, e di più tra la testa, ed il pariete superiore della cassa di legno trovarono una lastra di piombo lunga mezzo piede, e larga

\footnotetext{
${ }_{13}$ Il privilegio è ricordato nella conferma di Callisto II del 1123 (KEHR, Italia Pontificia, V, pp. 195-197).

${ }^{14} 27$ dicembre del 996: una serie di beni, tra i quali i castelli di Maneggio (Castelgugliemo secondo Castagnetti riportato da Tognana 2013, p. 113), Lendinara con la chiesa di Santa Sofia, Montagnana e Merlara, tutti indicati come curte domnicata cum castello et ecclesia inibi consistente (Annales Camaldulenses, I, app. n. 57; CDP, I, n. 76); 24 novembre 997 (Annales Camaldulenses, I, app. n. 59). Il 23 dicembre del 996, con atto sottoscritto nella chiesa della Vangadizza, il marchese Ugo dona, con divieto di alienazione e con la clausola che nel caso avesse avuto un figlio o una figlia a loro sarebbe spettata la proprietà, casa et curte domnicata cum castello e con la giurisdizione di Merlara; casa et curte illa domnicata cum castello et ecclesia di Montagnana con le pertinenze; casa et curte illa domnicata cum castello et ecclesia seu ripis et porticis di Lendinara; casa et curte illa domnicata cum castello et ecclesia di Maneggio con le pertinenze; infine beni presso la chiesa di Longula, a Rudicho, Conclum, Valli, Villa Maniana e Villa Archueta (Annales Camaldulenses, App. I, n. 57). Un anno più tardi, con atto rogato a Pisa il 20 novembre 997, acquista dalla sorella Waldrada allora in punto di morte, per il prezzo simbolico di un butericum aureum, la casa et curte domnicata seu castello di Vangadizza, con i molini e cum omnibus casis et rebus sive ripa juxta fluvium Adese, cui coheret ei ex una parte suprascriptum fluvium Adese, ex alia parte ... terra ecclesie Sancti Zenonis, ex tertia parte ... rivus qui dicitur Viba, ex quarta parte terra ecclesie monasterio. Nello stesso giorno cede al monastero il castello unitamente ad altri beni ubicati nella valle di Almerico nei pressi della valle di San Giorgio, beni probabilmente pervenutigli direttamente da Almerico II (Annales Camaldulenses, App. I, nn. 58-59; cfr. E. ROSSINI, Ugo "gratia Dei Gloriosissumus dux et marchio" e il monastero della Vangadizza, "Atti e Memorie del Sodalizio Vangadiciense", II, 1974-1981, pp. 3-25, a pp. 10-11).

${ }^{15}$ I corpi dei santi Primo e Feliciano, martiri romani sotto Diocleziano, dapprima custoditi sulla via Nomentana (al quindicesimo miglio), furono trasferiti dal papa Teodoro (642-49) nella chiesa urbana di Santo Stefano Rotondo, dove tuttora si trovano. Reliquie dei due santi erano state donate da papa Sergio II ad Eremperto, misso dell'imperatore Lotario, che le depose nella cappella a loro dedicata, da lui fondata agli inizi del IX secolo a Leggiuno, presso Milano. Non sappiamo se alla Vangadizza siano arrivate da Roma o da Milano. Nella ricognizione, effettuata nel 1617, in un loculo dentro l'altare a loro dedicato, vennero trovati due teschi "in due balle di vetro (G. BRONZIERO, Istoria delle origini e condizioni dei luoghi principali del Polesine di Rovigo, Venezia 1748, p. 171). Nel 1810 le reliquie sono state traferite nella parrocchiale di San Giovanni.

${ }^{16}$ A.E. BARUFFALDI, Badia Polesine (V). Lapidi ed iscrizioni, Saronno 1909, pp. 7-8.

${ }^{17}$ Anno MCCXXIII in eadem ecclesia Vangaticiensi sub Orlando abate, restaurata forte basilicae parte, reperta fuerunt corpora...: Annales Camaldulenses,

II, p. 390; IV, p, 293, con correzione della data 1223 in 1226.

${ }^{18}$ Annales Camaldulenses, II, n. 148.

${ }^{19}$ Vita auctore coevo, Acta Sanctorum Junii, Venezia 1572.

${ }^{20} \mathrm{G}$. BRONZIERO, Istoria delle origini..., p. 167.

${ }^{21} \mathrm{G}$. BRONZIERO, Istoria delle origini..., p. 171.
} 
cinque dita, da un lato della quale in lettere non molto ben scritte si leggeva: Hoc est corpus Beati Theobaldi, e dall'altro in caratteri di miglior forma era notato: Hoc est Corpus Sancti Theobaldi confessoris".

\section{LE DONAZIONI Di GUELFo IV E DI ALBERTo Azzo II}

Il 17 giugno del $1073^{22}$, Guelfo IV, figlio di Alberto Azzo II e duca di Baviera dal 1070, pro anima sua, della defunta madre Cunizza e di tutti i parenti, dona, con il consenso del padre, al monastero, retto dall'abate Pietro, due chiese, dedicate a Santa Maria e San Pietro, con tutte le loro pertinenze, ubicate ad Albaredo, porto sull'Adige in territorio vicentino, nonché la cappella dedicata a San Salvatore e Santa Maria a Verona con tutte le sue pertinenze in città, in Valpantena e in Valle Forte, a Varano e Porcile. La chiesa, ubicata in Corte Regia, era stata fondata da Berengario I presso l'Adige ${ }^{23}$, vicino al porto fluviale, il Ponte delle Navi. Rimarrà al monastero fino almeno alla fine del XII secolo ${ }^{24}$. La donazione di Guelfo assicurava al monastero due importanti punti di appoggio lungo l'Adige, in territorio vicentino (Albaredo) e in città.

Due anni più tardi, il 22 settembre del 1075, nel documento che ricorda la presenza del corpo di s. Teobaldo, Alberto Azzo II, pro mercede dell'anima sua e della moglie Cunizza, sepolta presso il monastero, dona beni per assicurare sumptum et stipendium monachorum ibidem servientium vel qui ibidem fuerint ordinati. La donazione, oltre a confermare le due chiese di Albaredo, comprende alcune paludi ubicate nel comitato padovano ${ }^{25}$.

Le due donazioni appaiono strategiche nel rafforzare economicamente il monastero nei due settori chiave: lo sfruttamento delle paludi, da trasformare in campi coltivati, e i commerci lungo l'Adige.

\section{LA SEQUENZA DELLA CHIESA IN BASE ALLE FONTI SCRITTE}

Le fonti scritte dichiarano esplicitamente tre differenti fasi istituzionali: 1. la fondazione della chiesa collegiata, prima del 955, ad opera di Almerico II e della moglie Franca; 2. una prima trasformazione in monastero ante 961; 3 . una rifondazione del monastero, nel 993, secondo l'ordine di san Benedetto (ad monasterium ibidem faciendum et construendum seu edificandum). Non precisano però quali opere architettoniche questa evoluzione istituzionale abbia comportato, anche se la fondazione, prima del 955, deve aver evidentemente comportato la costruzione della chiesa e di un annesso per ospitare il collegium di sacerdoti.

Un quarto rinnovamento architettonico, anche questo non esplicitamente dichiarato, è plausibile in base alla concatenazione, non credo fortuita, di eventi che si svolgono tra 1073 e 1075: la proclamazione a santo, prima del giugno del 1073, di Teobaldo; la rapida composizione della Vita ad opera dell'abate Pietro (abate nel giugno del 1073); il trasferimento del suo corpo all'interno della chiesa prima del settembre del

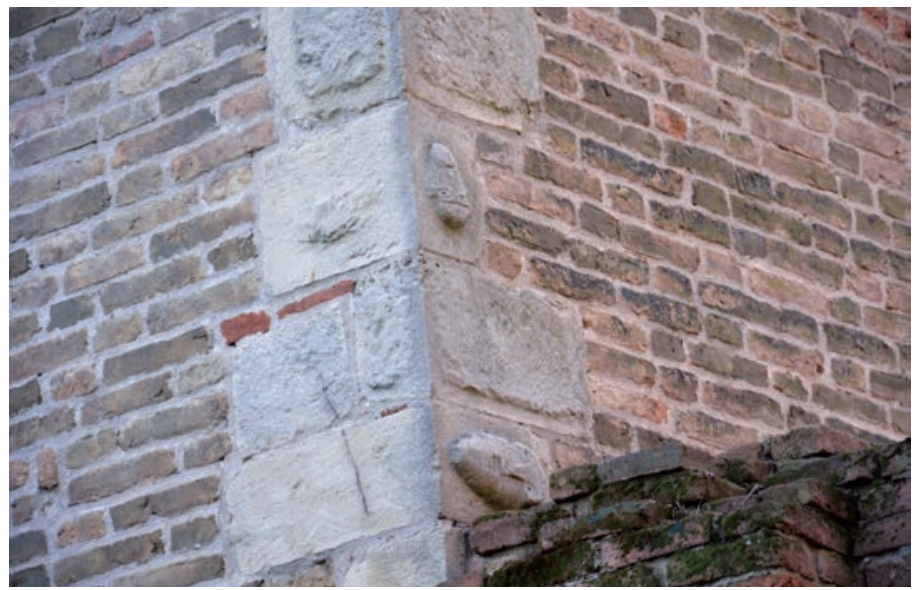

Fig. 3. Vangadizza, Santa Maria. Iscrizione MAGISTER PLACENT(inus) FECIT HOC (o)PUS, su un concio d'angolo del campanile.

1075; le due donazioni, entrambe sottoscritte in loco Este, dapprima di Guelfo IV, figlio di Cunizza, due anni più tardi da Alberto Azzo II, che allude al mantenimento dei frati presenti e futuri. Tutto sembra legarsi in una strategia di rilancio, economico e religioso, del monastero, orchestrata, tra 1073 e 1075, forse per iniziativa di Guelfo IV, dal 1070 duca di Baviera e dunque personaggio preminente della famiglia che intende esercitare tale ruolo anche nei possedimenti estensi. Questa ipotesi sembra confermata dai resti materiali della chiesa che suggeriscono un importante rinnovamento architettonico, probabilmente in concomitanza con la deposizione di san Teobaldo.

\subsection{I resti materiali}

Della chiesa della Vangadizza, integra fino al XIX secolo, sopravvivono ora solo alcuni resti in alzato che permettono di definirne la pianta a tre navate con le absidiole laterali a nicchia semicircolare all'interno di un rettangolo in muratura e una grande cripta ad oratorio. Ne conosciamo probabilmente anche l'architetto, un magister Placentinus (MAGISTER PLACENT(inus) FECIT HOC (o)PUS) ricordato in un'iscrizione graffita su un cippo romano collocato nell'angolo nord ovest del campanile (fig. 3), costruito assieme alla chiesa di seconda fase. Sulla base della pianta pubblicata in Internet sembra però che questa chiesa abbia semplicemente prolungato verso occidente un precedente edificio a pianta cruciforme, con ogni probabilità la collegita fondata da Almerico II prima del 955.

Prima di proporre una datazione della chiesa a tre navate, è necessario descrivere dettagliatamente quanto si conserva in alzato.

La parte bassa della facciata (fig. 4), ha paramento in laterizi (alcuni sono mattoni lidii romani di grandi dimensioni) e conci squadrati di reimpiego, in prevalenza di arenaria gialla (delle cave di Verona?), salvo alcuni frammenti di lastre di marmo proconnesio e di calcare impiegati nel prospetto, senza un ordine, e come rinforzo alle angolate.

\footnotetext{
${ }^{22}$ G. Annales Camaldulenses, II, n. 143; CDP, I, n. 219.

${ }^{23}$ DD Berengarii I, n. 97; CDV, n. 130.

${ }^{24}$ E. ROSSINI, La corte regia di Berengario e il monastero della Vangadizza (la donazione di Guelfo duca di Baviera, "Atti e Memorie del Sodalizio Vangadiciense", I, 1972-1973, pp. 307-315, a pp. 311-315.

${ }^{25}$ La donazione (Annales Camaldulenses, II, n. 148) comprende la decima parte della Valle del Termine a Vighezzolo e delle paludi dell'Argine della Mota a Palso; una parte di due porzioni della palude di Cavadicia a Megliadino; la decima parte di quella di Zudolegna ad Altaura che ora viene lavorata; la decima parte del lago Frondado a Casale e di Malauteda a Merlaria.
} 

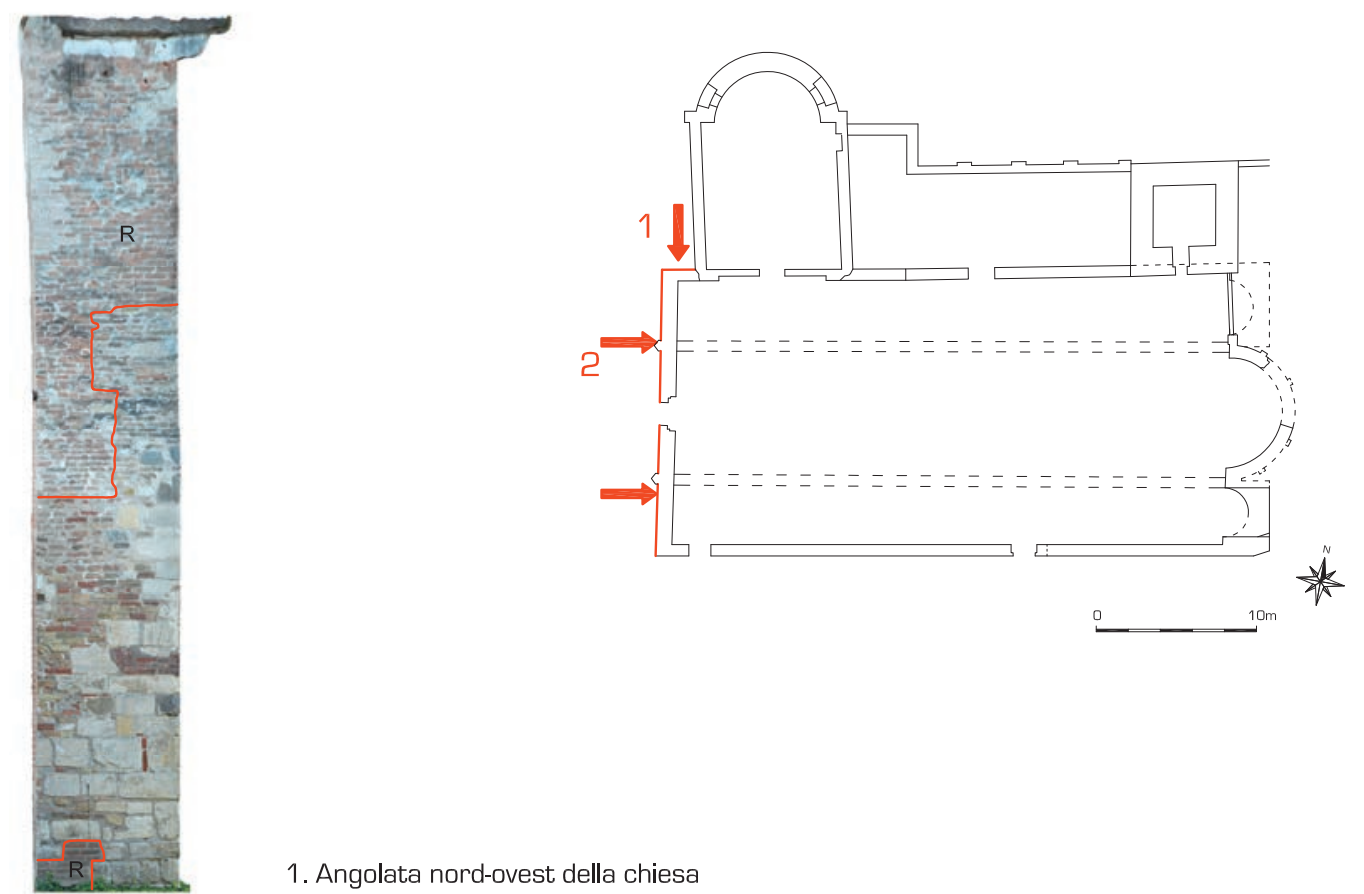

1. Angolata nord-ovest della chiesa

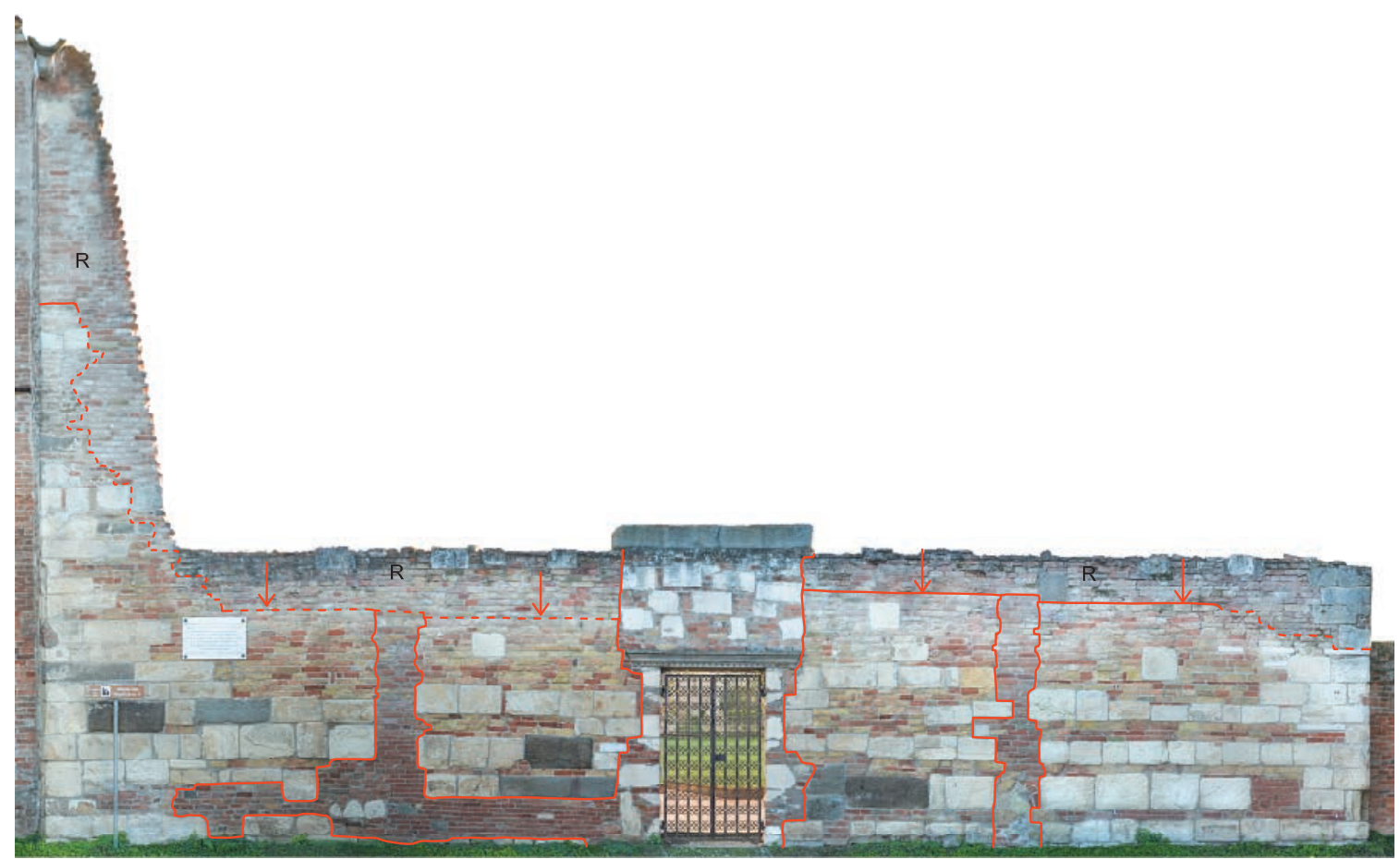

2. Facciata della chiesa

Fig. 4. Vangadizza, Santa Maria, facciata. Si notano la posizione delle lesene ed il profilo della porta originale.

I materiali sono posti in opera con una certa regolarità e i giunti, di malta con sabbia fine, erano in origine marcati da sottili stilature. Ai due contrafforti esterni, con fondazioni pentagonali, si contrapponevano internamente semicolonne su fondazioni rettangolari, a rinforzo dei divisori tra navate. Al centro della facciata vi è ancora qualche traccia della porta originaria, larga m 1,92 e con l'attacco di un arco in laterizi.

Il perimetrale sud (figg. 5-6), interamente conservato perché perimetrale del chiostro, ha paramento prevalentemente in laterizi, nel quale si riconoscono: circa al centro, lo stipite e un tratto di arco in laterizi di una porta (anteriore a quella del XVI secolo che l'ha sostituita); verso la facciata, un secondo arco con stipiti riferibile ad altra apertura; alcune nicchie (o aperture) tamponate; in alto, almeno tre finestre rettangolari tamponate.

Del perimetrale nord si conservano due brevi tratti: il primo (fig. 7), verso la facciata, è tagliato da una cappella del $\mathrm{XV}$ secolo ed ha paramento in laterizi e pietre di reimpiego disposte disordinatamente, il secondo (fig. 8), in corrispondenza del campanile, ha paramento in laterizi ed è tagliato dallo stipite di una seconda cappella laterale della quale, sul lato sud del campanile, si vede l'imposta del soffitto voltato; 

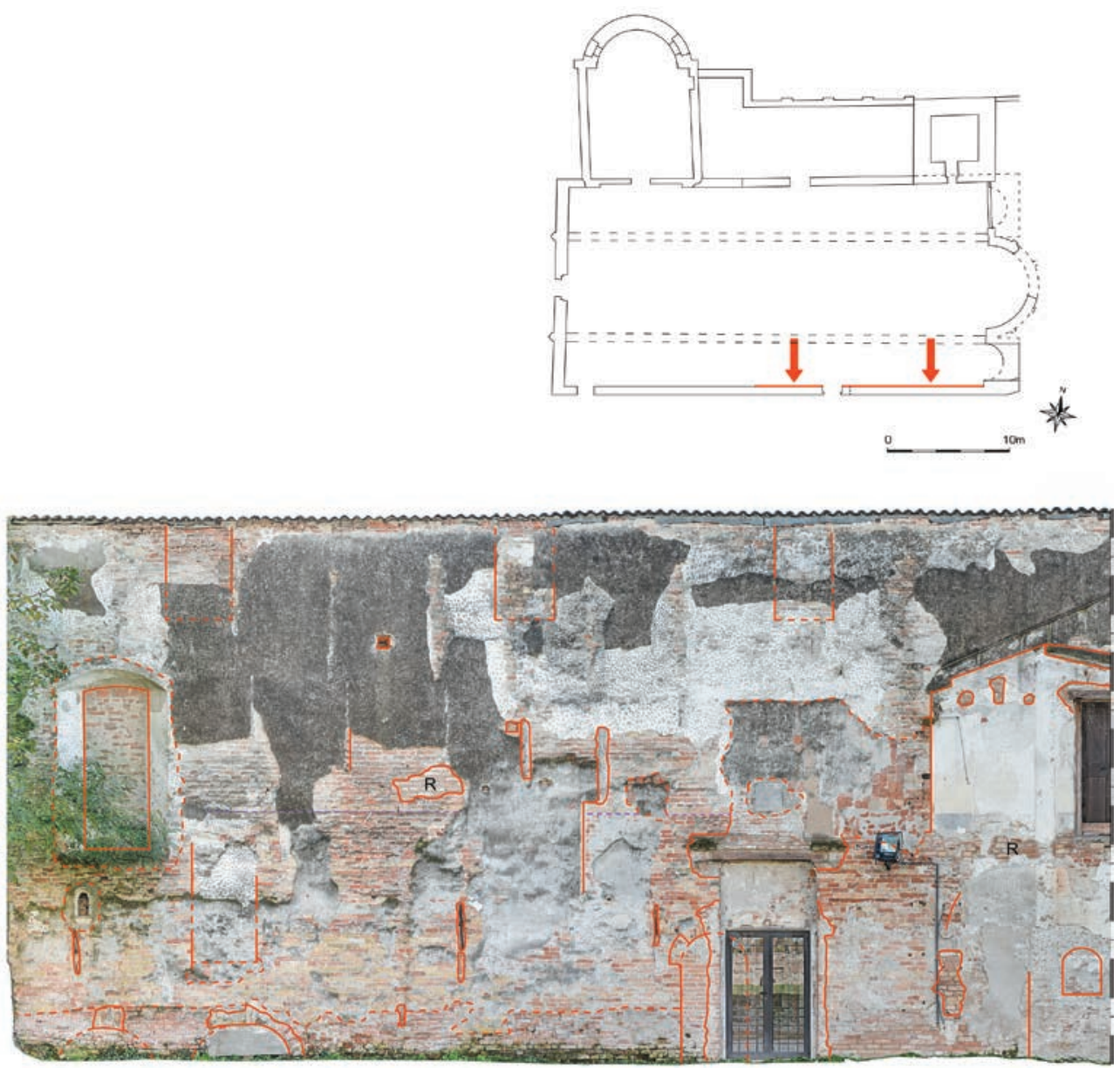

Fig. 5. Vangadizza, Santa Maria, tratto verso est del perimetrale sud. Si notano: in basso a sinistra gli archi della cripta, al centro la porta e in alto tre finestre tamponate.

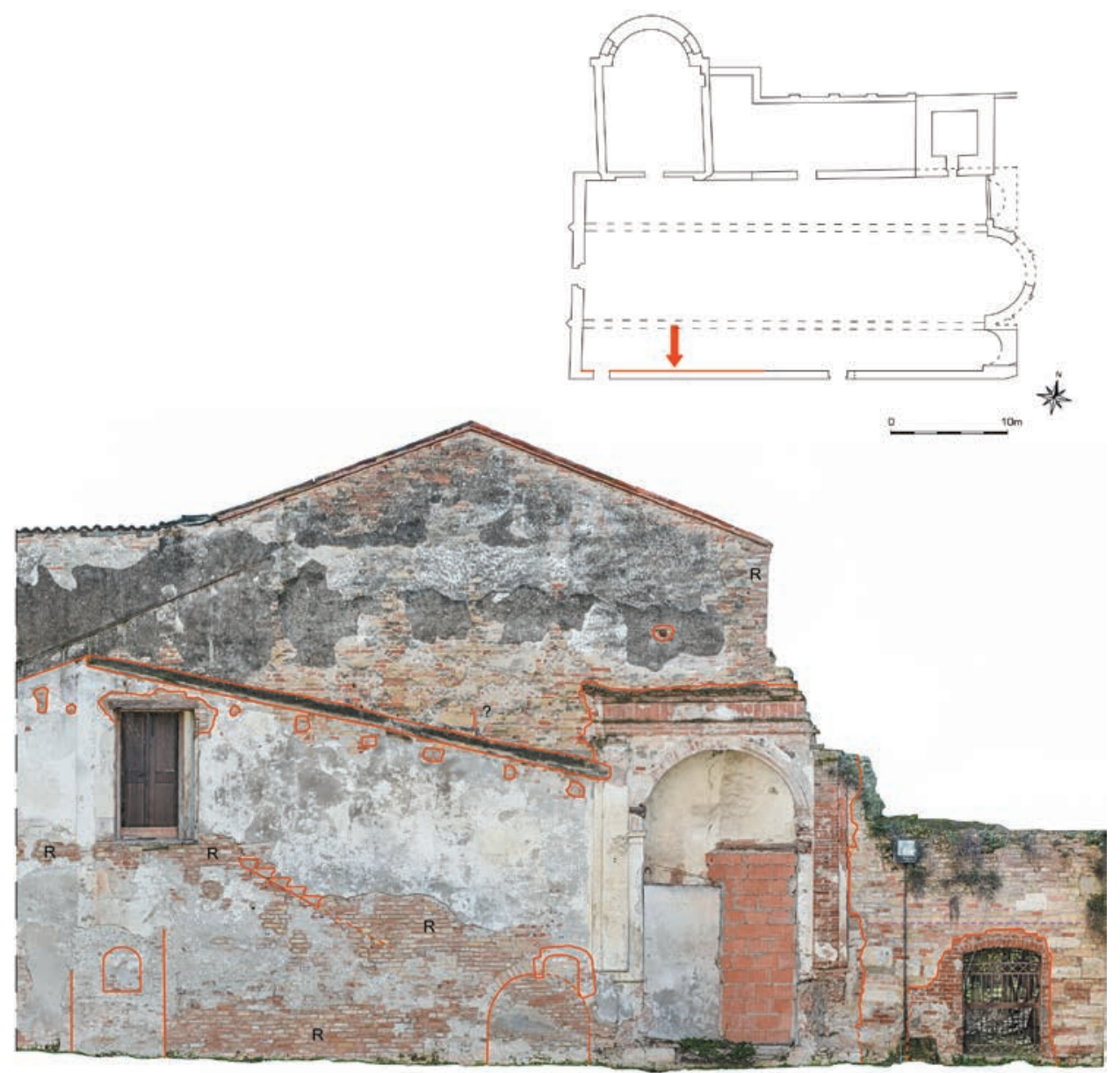

Fig. 6. Vangadizza, Santa Maria, tratto verso ovest del perimetrale sud. Si notano: il profilo di due aperture e il profilo di un edifcio addossato. 

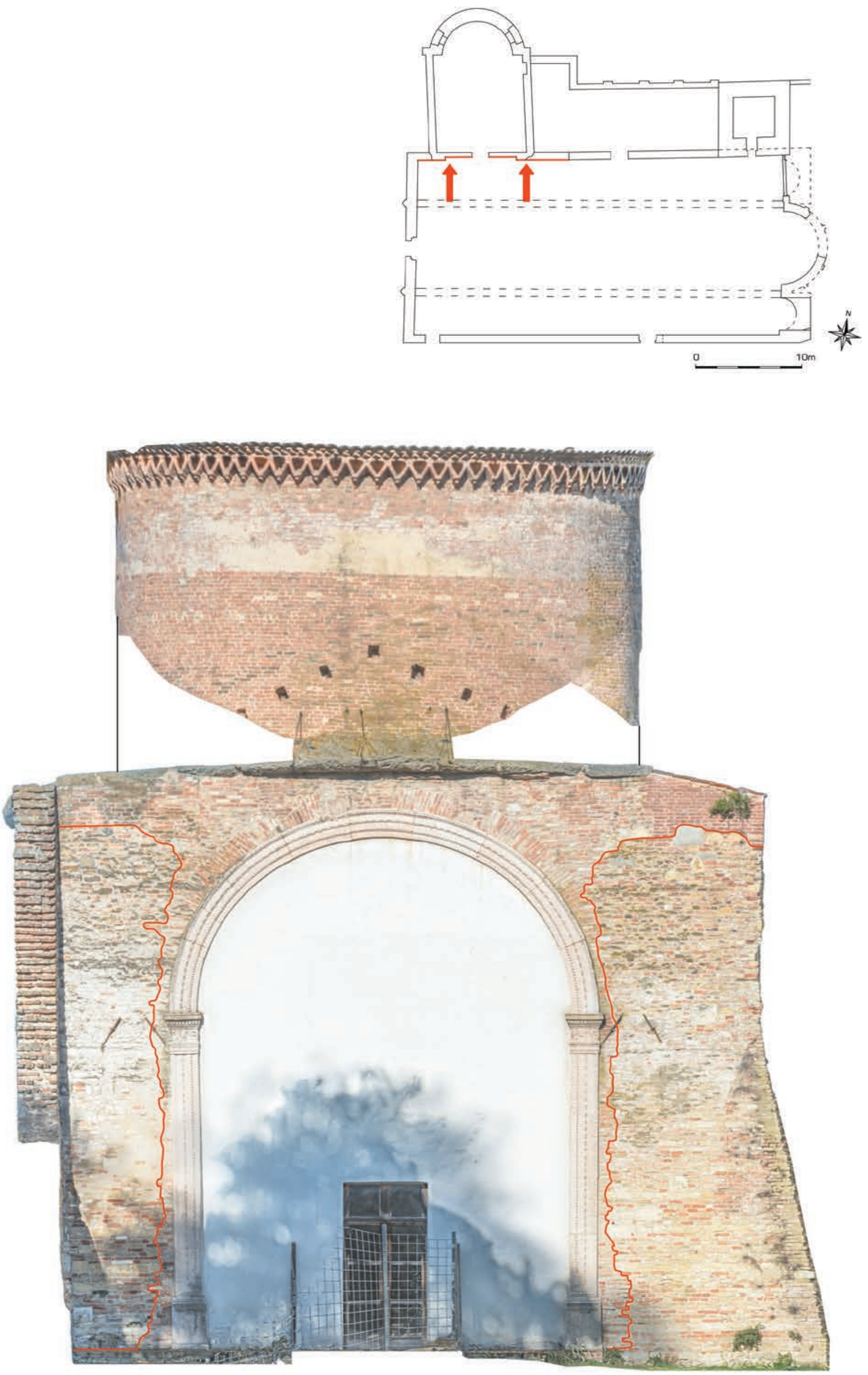

Fig. 7. Vangadizza, Santa Maria, tratto verso ovest del perimetrale nord, tagliato dalla cappella cinquecentesca. 


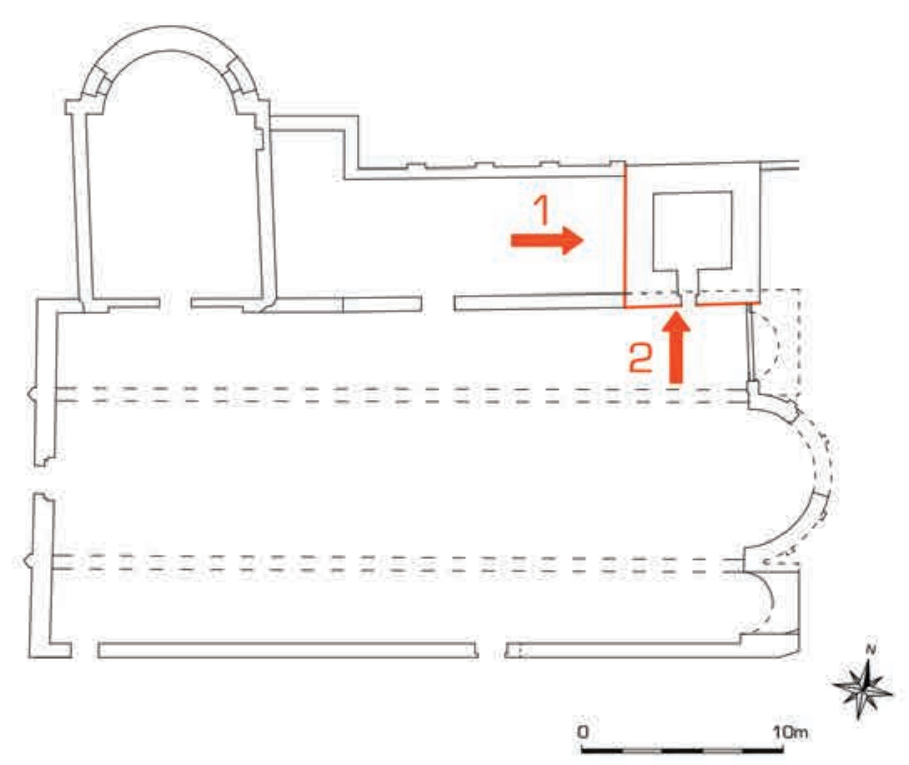

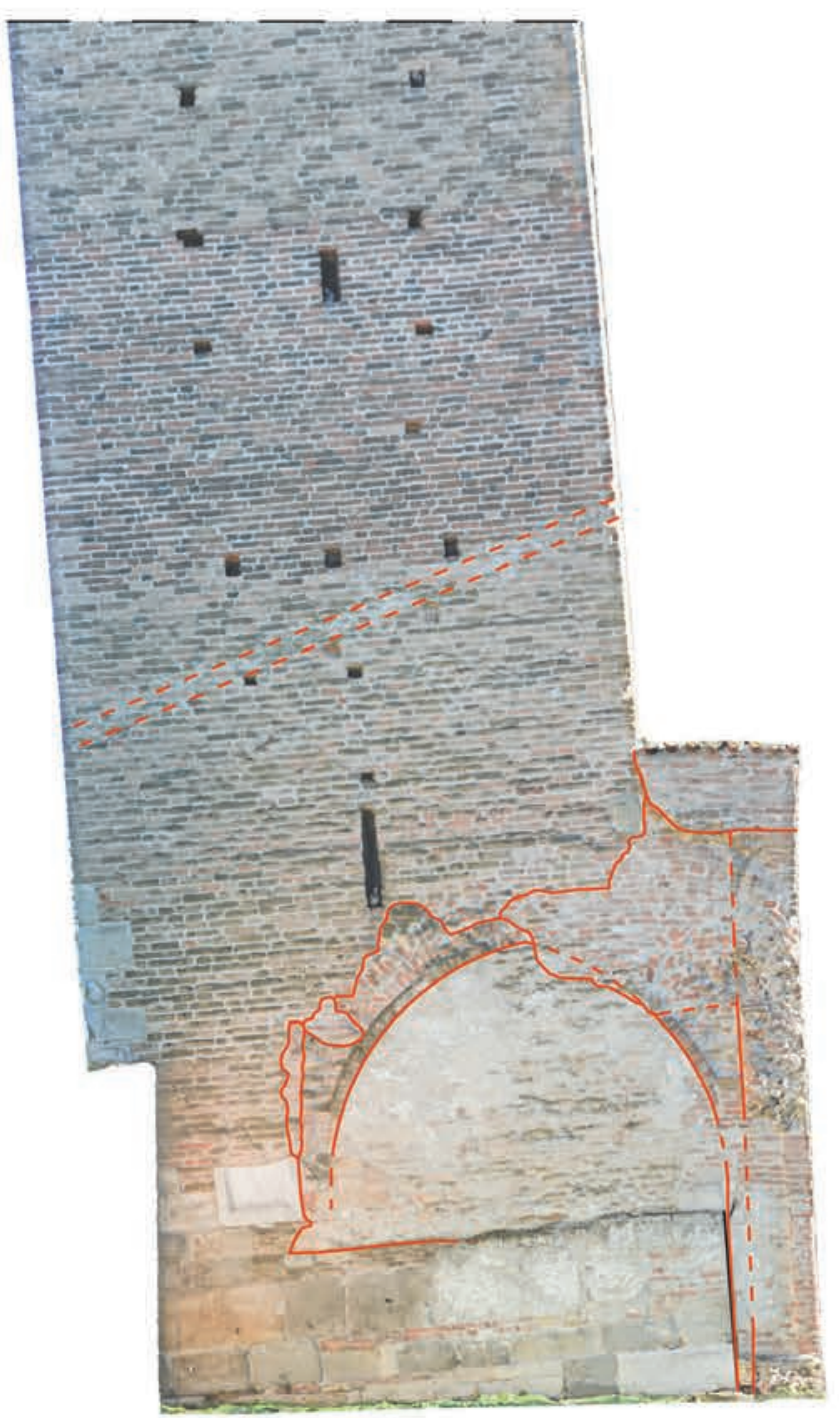

1. Parete ovest del campanile

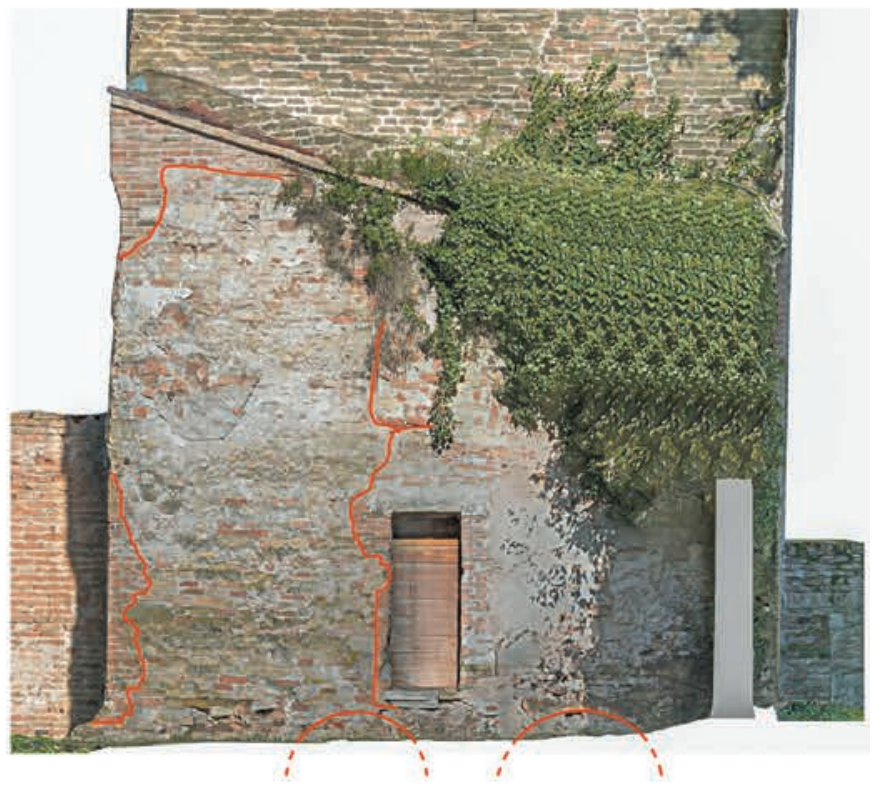

2. Parete sud del campanile, parete del perimetrale nord della chiesa

Fig. 8. Vangadizza, Santa Maria, parete ovest del campanile al quale si addossava una cappella e tratto verso est del perimetrale nord della chiesa (e base est del campanile). Si vedono, in basso, due arcate della cripta. 


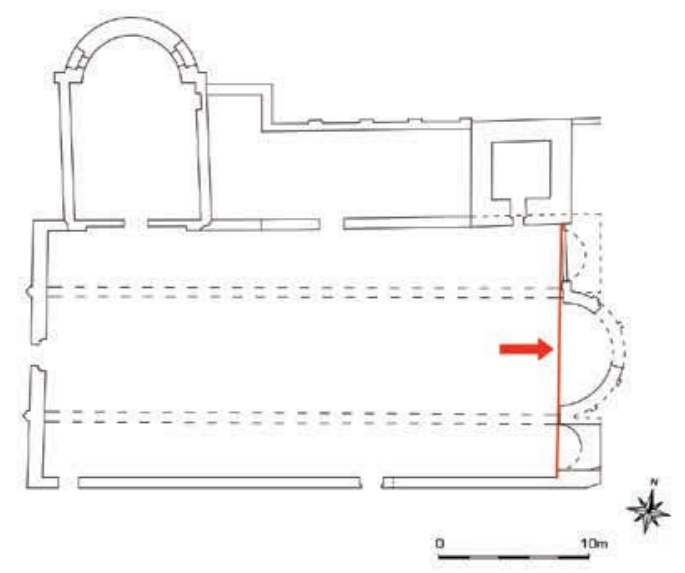

lesene; due sono nella parete ovest, in corrispondenza del campanile, altrettante sul lato opposto. All'interno delle archeggiature vi erano finestre, due delle quali sono riconoscibili (fig. 10). Questi elementi, integrati con quanto si osserva dalla pianta pubblicata in internet, suggeriscono una cripta, estesa su $137 \mathrm{mq}$, a pianta cruciforme e con volte a vela.

Alla chiesa, sul lato nord, si addossava un campanile di $\mathrm{m} 6,32$ x 6,10 (fig. 11), del quale si conserva solo la base in blocchi di pietra (alcuni dei quali ricavati da monumenti funerari romani, la base di una colonna, un cippo parallelepipedo decorato, una mensa d'altare paleocristiana, qualche rilievo antropomorfo erratico altomedievale). Dopo un crollo, il campanile è stato ricostruito riutilizzando i laterizi romani; infine è stata innalzata la cella campanaria nella quale è inserita una pietra con data 1517.

Il lato sud della chiesa fungeva da perimetrale del chiostro, nel quale si riconoscono almeno un paio di edifici medievali. Orientati nord sud, avevano piani di calpestio più bassi di almeno un metro rispetto agli attuali. Lo si deduce dalla quota dell'intradosso (pari a m o,83 rispetto al pavimento più recente) di una porta larga $\mathrm{m} \mathrm{o,87}$ nel corpo di fabbrica ovest e di una seconda con quota sempre all'intradosso di $\mathrm{m}$ 1,37 in quello est. Sono riferibili al chiostro del monastero, ricordato nel $1123^{26}$ e riorganizzato nel XV secolo

Fig. 9. Vangadizza, Santa Maria, perimetrale est, con l'abside laterale ovest tamponata e quella centrale nella quale si vedono una finestra tamponata e gli archi della cripta.

L'abside centrale (fig. 9), costruita quasi interamente in laterizi di grandi dimensioni senza un nucleo, sopravvive parzialmente. In alto, è stretta tra muretti verticali contro i quali si addossavano gli spioventi della navate laterali. Esternamente la scandivano lesene rettangolari, di cui una sola, di cm 14 X 41 x 18, è conservata. Anche delle tre finestre ad arco a tutto sesto ne rimane una soltanto.

Sono riferibili all'intervento del 1335 un ampliamento nel settore centrale e una parziale rifoderatura. Si riconosce la fondazione a sacco di un grosso pilastro (tra abside centrale e abside nord) che sorreggeva lo stipite dell'arco trionfale, mentre, in sezione, si vede la traccia del pavimento dell'abside.

Le archeggiature cieche delle volte a crociera della cripta sono visibili sia nell'abside centrale, sia negli angoli orientali delle navate laterali: nell'abside, al di sotto del pavimento del presbiterio, se ne vedono solo tre (rispetto alle cinque ipotizzabili), inserite nei perimetrali e poggianti su nelle forme attuali.

\subsection{Dalla collegiata di Almerico II al monastero di Ugo e di Alberto Azzo II}

Nelle condizioni attuali di lettura del monumento, rimangono aperti importanti problemi, alcuni dei quali troveranno soluzione nella pubblicazione degli scavi. Nell'absidiola meridionale della chiesa si nota una preesistenza: un frammento di muratura con paramento in pietre spaccate di trachite, più antico e ha un orientamento diverso rispetto all'absidiola stessa. Ha il medesimo orientamento del corpo di fabbrica verso est che potrebbe corrispondere alla canonica del X secolo, con quote più basse di almeno un paio di metri rispetto alla seconda chiesa;

Anche alcuni elementi della seconda chiesa rimangono incerti: le absidiole laterali a nicchia semicircolare all'interno di un rettangolo in muratura; il variare degli spessori dei

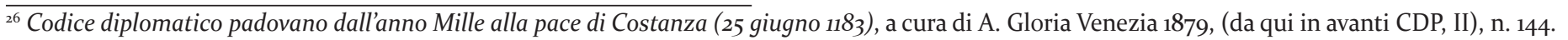




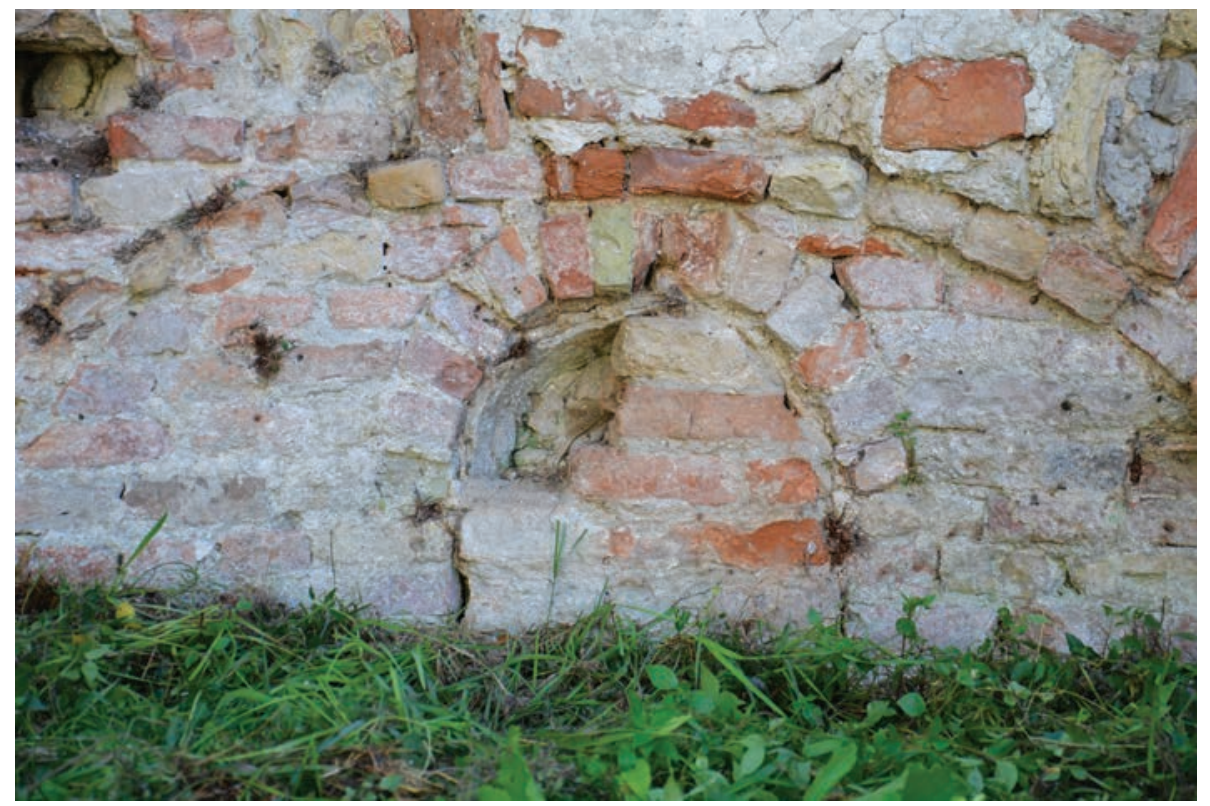

Fig.10. Vangadizza, Santa Maria, finestra tamponata al di sotto di un arco della cripta.

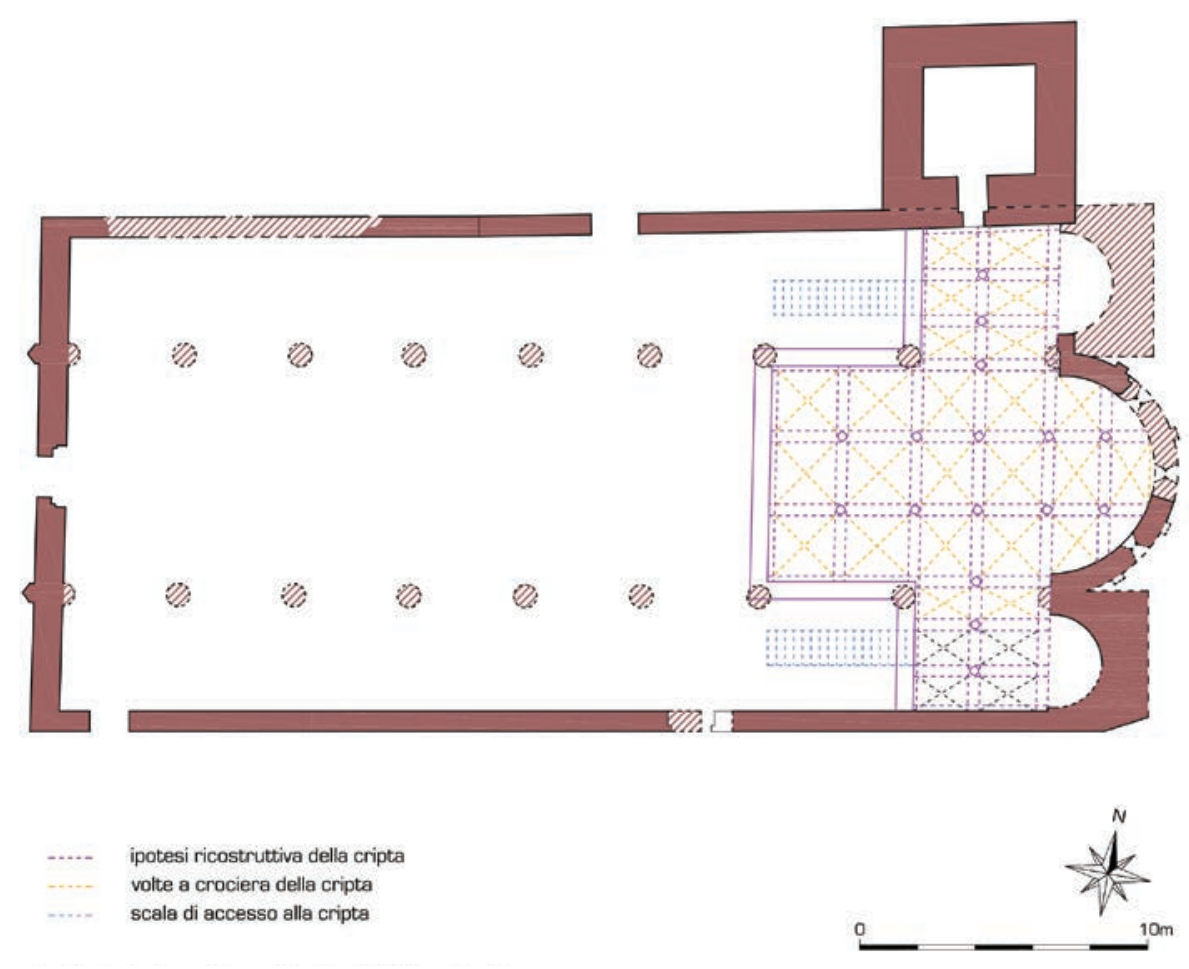

Fig. 12. Vangadizza, Santa Maria, ipotesi della pianta della seconda chiesa che sembra riutilizzare, come cripta, la prima chiesa con pianta a croce.

perimetrali della chiesa (un $\mathrm{m}$ in facciata contro i ca. $80 \mathrm{~cm}$ nell'abside e i 70 del perimetrale nord); la sequenza della parete meridionale della seconda chiesa, ricoperta in larga parte da un intonaco che ne impedisce una lettura stratigrafica; la funzione di un divisorio a 19 metri dalla facciata: delimitava lo spazio riservato ai monaci rispetto a quello dove avevano accesso i fedeli o è riferibile alla facciata della prima chiesa?

Pur con questi problemi aperti, in base ai dati materiali, credo si possano ipotizzare almeno due fasi costruttive (fig. 12):

(1) la chiesa fondata, prima del 955, da Almerico II, corrisponde probabilmente alla cripta cruciforme della seconda chiesa, salvo una maggiore estensione della navata, suggerita dal muro verso ovest. L'impianto cruciforme, se confermato,

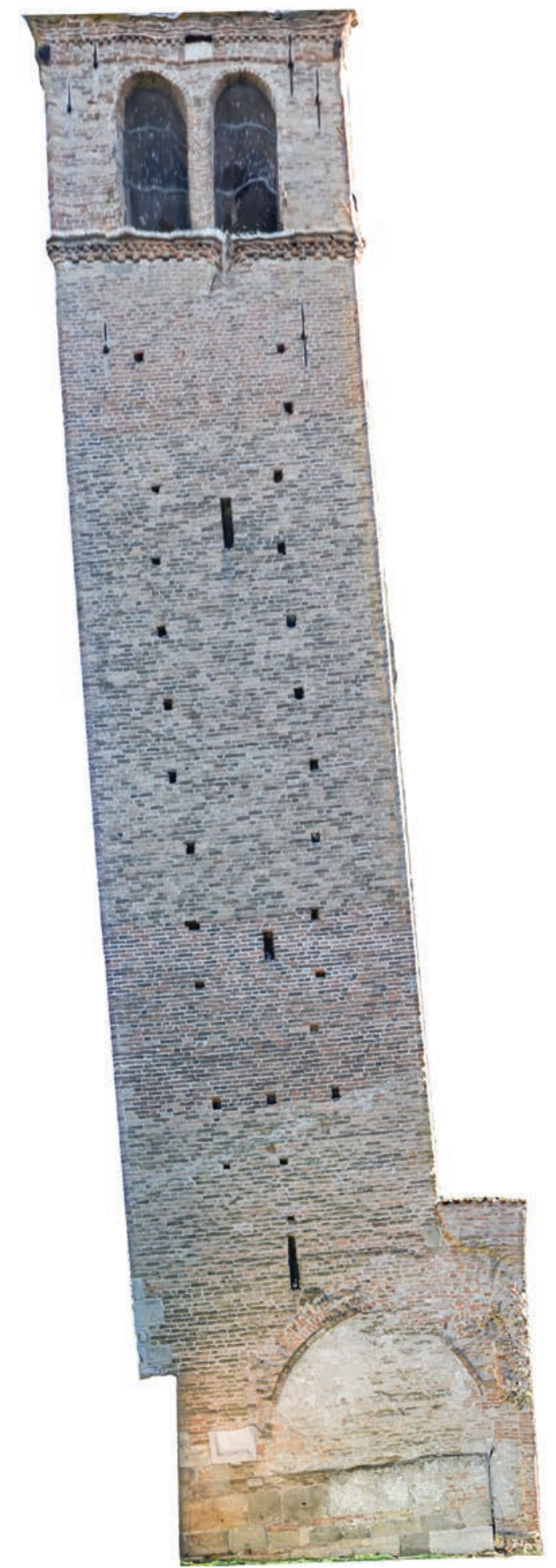

Fig. 11. Vangadizza, Santa Maria, lato ovest del campanile.

troverebbe confronti in chiese databili tra IX eX secolo, tutte legate ad una committenza alta, dal San Michele di Candiana (Padova), a San Pietro di Gazzo e San Zeno di Bardolino nel veronese, a San Giorgio di Quarazze in Alto Adige;

(2) la successiva grande chiesa a tre navate, larga $\mathrm{m} \mathrm{17,80}$ e lunga ca. 40 (misure esterne), con una superficie di mq 595 (comprese le absidi) al netto delle murature, trova confronti per la cripta ad oratorio (ma non per lo sviluppo cruciforme) nel vicino San Fidenzio di Megliadino (post 978). A quando si data questa fase? È conseguenza delle donazioni di Ugo il grande, esplicitamente finalizzate alla rifondazione di un monastero, senza però alcun accenno alle opere architettoniche, o degli interventi degli Obertenghi attorno al 1075? 
Per ora possiamo osservare che l'iscrizione del magister placentinus è stata datata "tra la fine dell'XI e gli inizi del XII secolo" 27 e che la base del campanile è simile a quella delle torri padovane assegnate alla fine dell'XI secolo (Chavarria 2011), ma questo non è dirimente perché troviamo la medesima tecnica in quella che si ritiene carolingia a Sant'Ambrogio a Milano. È vero però che i pilastri pentagonali in facciata hanno confronti nel San Lorenzo di Verona della fine del secolo XI e il contesto storico degli anni 73-75, sopra delineato, sembra adatto per avviare la rifondazione architettonica affidata al magister placentinus, proveniente da Piacenza, nei cui comitato i protoestensi possedevano beni ${ }^{28}$.

E tuttavia, a sostegno di una costruzione della chiesa al tempo di Ugo il grande, oltre al documento del 993, si può addurre anche che vi trovarono sepoltura, attorno al 1040 il marchese Ugo e, una decina d'anni dopo, Cunizza, molto prima, dunque delle donazioni di Alberto Azzo II e di suo figlio Guelfo IV.

\subsection{La chiesa mausoleo degli Obertenghi}

Un utilizzo della chiesa come mausoleo degli Obertenghi è testimoniato dalle fonti scritte nel 1040, quando il normanno Rodolfo, come si è visto, elargisce una donazione alla chiesa pro memoria del marchese $\mathrm{Ugo}^{29}$ e nel 1050 ca. quando vi viene sepolta Cunizza, moglie di Alberto Azzzo II. È però plausibile lo fosse fin dal tempo di Almerico II, almeno per tre motivi, nessuno dei quali probante ma che insieme ne offrono un'alta probabilità: (a) le due cospicue donazioni effettuate da Almerico II poco prima di morire e quella di Franca subito dopo la morte del marito; (b) la pianta cruciforme, che ha come lontano, e illustre, modello la chiesa dei Santi Apostoli a Costantinopoli che accolse le spoglie dell'imperatore Costantino e dei suoi successori; (c) la presenza di tre sarcofagi romani, uno dei quali venne utilizzato per Cunizza, dunque attorno al 1050.

\section{TRE SARCOFAGI}

Presso l'ex monastero della Vangadizza si conservano tre sarcofagi. Quello che si trova nel chiostro, privo di coperchio, ha la faccia principale ripartita da tre arcate (a tutto sesto le laterali, ribassata la centrale) innestate su quattro lesene con basi e semicapitelli. Le arcate incorniciavano rilievi che sono stati scalpellati al pari del fianchi del sarcofago. Datato alla metà del III secolo, trova confronti con il sarcofago di villa Bissarri a Sovizzo (Vi) e con quello proveniente da Santa Maria di Sandrigo, oggi nel porticato di palazzo Da Schio a Vicenza ${ }^{30}$.

Davanti alla facciata della chiesa sono stati rimontati su quattro pilastrini altri due sarcofaghi (fig. 2). Il primo ha pareti lisce ed è sormontato da un coperchio con i quattro

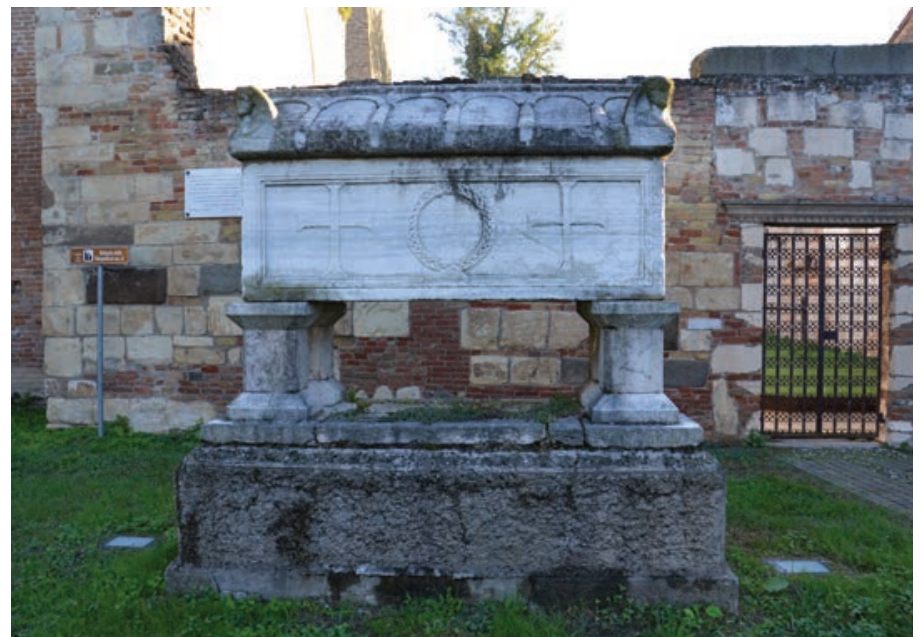

Fig. 13. Vangadizza, Santa Maria, sarcofago "a cassapanca" con la faccia principale ornata da due croci con bracci espansi e serto stilizzato centrale.

acroteri pertinenti ad un altro sarcofago. Il secondo (fig. 13) è del tipo "a cassapanca", con la faccia principale ornata da due croci con bracci espansi e serto stilizzato centrale e quelle laterali da una singola croce. Il motivo decorativo rimanda alla produzione ravennate di VI secolo, in particolare a sarcofagi a pannello e cornice continua modanata da Ravenna (a San Vitale, nel portico e nel cortile antistante l'ingresso). Probabilmente rilavorato con un motivo di sei archi, sostenuti da semicolonne con capitelli corinzi, è invece il coperchio con acroteri. Le sepolture in sarcofagi, sia romani e paleocristiani di reimpiego, sia nuovi ma ispirati ai modelli di VI secolo, sono testimoniate per tutto l'altomedioevo a Ravenna e nei centri Venetici lagunari. Anche nel padovano, sarcofagi altomedievali a cassa semplice sono testimoniati sia presso la chiesa di Santa Giustina di Padova, sia nel San Tommaso di Megliadino (infra). È dunque plausibile che il sarcofago ravennate della Vangadizza sia stato recuperato da Almerico II, i cui interessi a Brondolo e a Ferrara sono documentati dalle donazioni.

\section{LA TOMBA DI CUNIZZA E DEL MARITO ALBERTO AZZO II}

Alcuni lavori di ristrutturazione della chiesa, intrapresi nel $1335^{31}$ dall'abate Severo di Sassoferrato, richiesero, nell'agosto dell'anno seguente, l'apertura e lo spostamento di un'arca lapidea ${ }^{32}$. All'interno venne trovata un'epigrafe, scolpita in quodam matono cocto, che serviva come cuscino per la testa di un defunto, accanto al quale, con orientamento contrapposto, vi era un secondo scheletro. Entrambi erano avvolti in un panno ornato d'oro. Il testo dell'epigrafe, trascritto in un codice del monastero e pubblicato in due versioni leggermente diverse, ricorda le origini regali di Cunizza (figlia del duca guelfo Welfonte, imparentato con

\footnotetext{
${ }^{27}$ A. DIANO, La chiesa abbaziale di S. Maria della Vangadizza nel quadro dell'architettura medievale dell'entroterra veneto. Note preliminari, "Atti e Memorie del Sodalizio Vangadiciense", V, 1990, pp. 183-210, a p. 194.

${ }^{28}$ Confermati da Enrico IV, nel 1077, a Folco e Ugo, figli di Alberto Azzo II (DD Heinrici IV, n. 289).

${ }^{29} \mathrm{CDP}, \mathrm{I}, \mathrm{n} 140$.

${ }^{30}$ C. FRANZONI, N. DOLCI, Contributo allo studio dei sarcofagi pagani della bassa valle del Po, in Felix Ravenna, CXI, 1981, pp.11-16.

${ }^{31} \mathrm{Nel} 1335$ erano iniziati i lavori per la ricostruzione del coro: Anno... MCCCXXXV indictione tertia, reparata et reformata fuit haec ecclesia et chorus dicte ecclesiae factus fuit tertio per domnum Severum de Saxoferrato (Annales Camaldulenses, V, p. 356). La notizia è fornita da Giangirolamo Bronziero, che asserisce di averla tratta da un codice conservato nel monastero. Bronziero scrive tra 1628 e 1629, ma la pubblicazione è uscita oltre un secolo dopo.

${ }^{32}$ Il Bronziero trascrive, credo in modo sbagliato, quanto scritto nel codice: arca lapidea quae est revoluta cum fundo superius, ovvero il sarcofago capovolto con il fondo in alto.
} 
gli imperatori), la nobiltà e la ricchezza del marito Alberto Azzo II e si conclude con un anatema contro un eventuale violatore della tomba ${ }^{33}$. Sono da sottolineare la dimensione dell'epigrafe, proporzionata rispetto alla funzione di poggiatesta di Cunizza e dunque il suo particolare significato rituale. A differenza dei testi epigrafici incisi sui lati esterni dei sarcofagi o sulle lastre di copertura delle tombe o appese alle pareti destinati a perpetuare visibilmente il ricordo, questa è una sorta di lettera di presentazione da spendere nel giorno del giudizio finale, al quale spera di poter partecipare uscendo dalla tomba non violata da alcuno. I versi non erano destinati a conservare una memoria della defunta visibile dai fedeli, ma erano rivolti a chi aveva partecipato alla cerimonia (e non sappiamo se allora ne fosse stata fatta una pubblica lettura o commento) e soprattutto alla stessa Cunizza, della quale delineano la nobiltà del padre e della famiglia di origine, le doti morali (vir prudens magnusque) e la ricchezza del marito, senza confronti in Italia. Hanno dunque il medesimo significato delle iscrizioni dipinte all'interno delle tombe e dei corredi deposti nel corso del funerale. E l'autore, più che il marito, sembra la stessa Cunizza. Alla morte di Alberto Azzo II, nel 997, fu probabilmente Guelfo IV, figlio di Cunizza e potente duca di Baviera, ad organizzarne la sepoltura all'interno del medesimo sarcofago conservandovi l'iscrizione per la madre.
In conclusione, Santa Maria della Vangadizza fu il punto di riferimento, verosimilmente fin dall'origine come mausoleo, di importanti famiglie di funzionari imperiali i cui beni e la cui autorità, tra IX e XI secolo, si svilupparono tra la Toscana e il basso Adige. La seconda chiesa, di cui tratto, venne fondata, probabilmente poco prima del 1078, dagli Obertenghi che avevano spostato il centro del potere di queste famiglie dalla Scodosia ad Este.

\section{LA COLLEGIATA DI SANTA MARIA DI CARCERI}

Santa Maria di Carceri sorge in una posizione strategica, non lontana dal fiume Santa Caterina, nome del Guà-Frassine a sud di Este, e dai suoi affluenti, uno dei quali passava per Carceri. I beni di Santa Maria si trovavano in un comparto di relativamente alta pianura (10-12 m s.l.m.), tra gli insediamenti di Ponso e Vighizzolo, dove sono noti ritrovamenti archeologici dell'età del Ferro e romani ${ }^{34}$. Solo più sud di Carceri si estendeva un'area di bassura (m 4-5 s.l.m.), occupata, fin dall'antichità, dai laghi di Ponso e di Vighizzolo, la cui bonifica venne ultimata tra XVI e XVII secolo 35 . Anche attorno al monastero il disegno particellare dei campi tradisce una progressiva riduzione a coltura delle aree circostanti e la partecipazione attiva nella conquista di

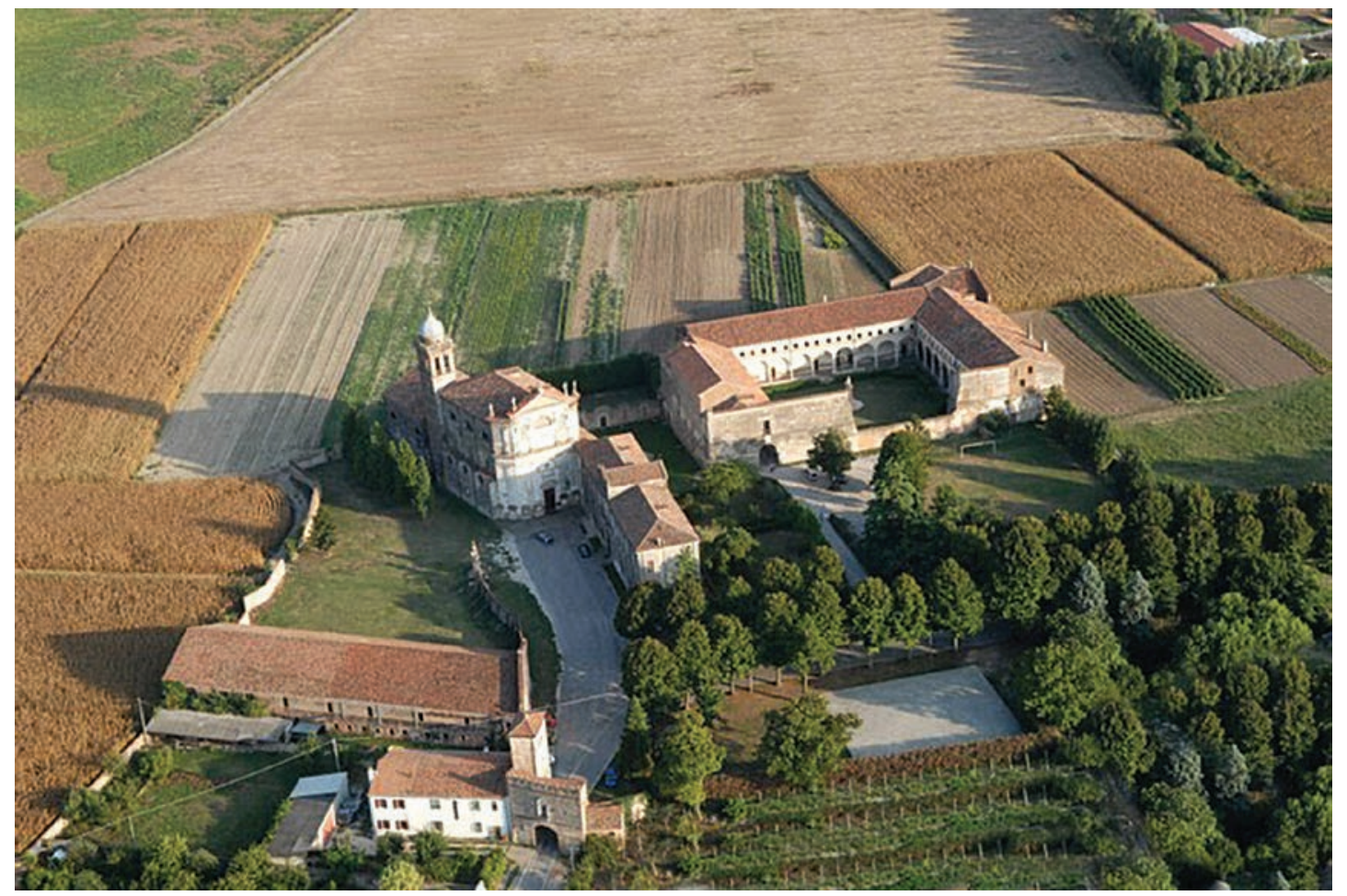

Fig. 14. Carceri, Santa Maria, veduta dall'alto.

\begin{abstract}
33 L'epigrafe, trascritta da G. Bronziero, Istoria delle origini, l p. 177), è stata ripubblicata dal Campagnella e dal Muratori, infine dal Baruffaldi con alcune varianti. Il Bronziero, che asserisce di "aver trascritto fedelmente quei versi dall'autentico, niente aggiongendo, né minuendo", così la riporta: Acta GUNIGULDIS regali stemate fulti./ Indole nobilior nullus in orbe fuit./ Germine. VAlfontis magni sum natu Hemani/ Induperatorum iste fuit titulus/ Vir meus egregius popul lucupletior ullus/ Haud manet Italia haud simile peperit,/ Azo, vir prudens magnusque, Marchio fulget/ Quem credo memorem semper adesse mei/ In terra coelestis sobolem concessit utrique/ qui WHElfons dictus duxque potensque pius./ Hunc violare nefas loculum, nec inde recedam donec vera carne resurgam. Aggiunge poi. "Non nego però che ove si legge Acta, non possa chi scrisse aver voluto dire Victa ... e dove è detto Hemani, si abbia voluto dire Germani, perché forse nel mattone saranno dal tempo state guastate quelle lettere" (G. BRONZIERO, Istoria delle origini..., p. 177). La versione del Baruffaldi è diversa: Victa Guniguldis regali stemmate fulsi. Indole nobilior nullus in orbe fuit germine. Welfontis magni sum nata germana. Induperat ... iste fuit titulus vir meus egregius populus lucupletior ullus haud manet Italiae haud simile peperit: Azo, vir prudens magnusque, Marchio fulget quem credo memorem semper adesse mei in terra, celestis sobolem concessit utrisque qui Welfontis dictus duxque potensque pius. Hunc violare nefas loculum ne inde recedam donec vera carne resurgam (A.E. BARUFFALDI, Badia Polesine..., pp. 5-7).
\end{abstract}

${ }_{34}$ Carta archeologica del Veneto, Rovigo, 163-170.

35 M. VIGATO 1997, Il monastero di S. Maria delle Carceri..., pp. 16 e 98-108. 
nuovi spazi agrari già in corso alla fine del X e proseguita anche dopo il Mille.

In queste fasi pionieristiche le chiese sono avamposti in grado di attrarre nuova popolazione e di creare, al contempo consenso sociale.Tali funzioni traspaiono nella rifondazione della chiesa di San Tommaso di Megliadino, in una zona non lontana da Carceri ${ }^{36}$. Con un privilegio, emanato nel 978, Gauslino, vescovo di Padova, al fine di ricostruire la chiesa distrutta dagli Ungari, concede ai presbiteri, ai diaconi e ai servi della chiesa le decime dei novalia di Megliadino e Saletto. Proventi da impiegare nella costruzione degli annessi, del cimitero e per impiantare una vigna, lavori nei quali sono impegnati con denaro ed opere, l'arciprete Zenone e cinque presbiteri37.

Santa Maria alle Carceri compare per la prima volta in due rogiti notarili del $1078^{38}$. Non sappiamo però se si riferiscano ad una chiesa già esistente o in costruzione. La sua fondazione è stata attribuita dal Muratori alla famiglia di Alberto Azzo II ${ }^{39}$, anche se dai documenti emerge un loro interessamento solo più tardi, grazie a due documenti del 4 ottobre del $1117^{40}$. Con il primo, un placito tenuto juxta Sanctam Teclam in villa que vocatur Este, Enrico il Nero, pronipote di Alberto Azzo II e duca di Baviera, concede la sua protezione ${ }^{41}$; con il secondo gli dona una Braida ${ }^{42}$.

I due rogiti del 1078 attestano l'acquisto di terre nel veronese, ben lontano dal luogo dove si trovava la chiesa, interessi difficili da gestire senza l'appoggio di Alberto Azzo II, massima autorità pubblica della zona. E dunque plausibile l'ipotesi del Muratori che la fondazione sia da mettere in relazione con lo spostamento degli interessi dei Protoestensi ad Este. In questa strategia erano necessari non solo una sede di prestigio, realizzata con la costruzione di una nuovo castello sulla vecchia fortificazione del VI secolo, ma anche un'istituzione religiosa di prestigio, non lontana da Este e in un'area di nuova conquista agraria.

Nella fortuna della chiesa, dopo la morte del marchese, hanno giocato un ruolo anche i cattivi rapporti tra il duca di
Baviera, figlio di Cunizza, e i fratellastri, figli della seconda moglie, entrambi interessati al controllo delle proprietà nella giudicaria di Monselice. A sostenere la strategia del ramo bavarese, viene coinvolto anche Sinibaldo, cui si deve ${ }^{43}$ il rafforzamento della chiesa di Carceri con la tutela vescovile e la sostituzione dei canonici secolari con quelli agostiniani, riformati secondo la regola di Santa Maria in Porto di Ravenna ${ }^{44}$. Decisione che li mette al riparo dalle ingerenze del vescovo.

Con questo duplice appoggio, la canonica, nel corso dell'intero XII secolo, aumenta il suo prestigio ricevendo nuove consistenti donazioni dai marchesi, dai vescovi, in particolare da Bellino e Gerardo, e dai loro vassalli. A coronamento di questa ascesa, nel 1177, l'imperatore Federico Barbarossa e il papa Alessandro III concedono la loro protezione ${ }^{45}$. Effetto di questa nuova autorevolezza, oltre all'accumulo dei beni ${ }^{46}$, sono la crescita della comunità e il rinnovamento architettonico del complesso. Avviato nel 1168 con la richiesta, inoltrata dal priore e dai canonici, di poter utilizzare le pietre di Casale, viene completato in una ventina di anni. Nel 1189 il patriarca di Aquileia Gotifredo, affiancato dai vescovi di Padova, Vicenza e Belluno, riconsacra solennemente la chiesa alla presenza del marchese Obizzo, di Lamberto e Odone di Menegelva. In quell'occasione tutti i partecipanti, vescovi e laici, elargiscono una donazione ${ }^{47}$.

Gli atti notarili, già prima della riconsacrazione del 1189, vengono redatti nell'ecclesia e nell'hospitalis (1165), indizio di un complesso monastico articolato che assicurava anche l'accoglienza particolarmente sentita nella regola dei portuensi ${ }^{48}$. In quelli successivi compaiono, senza che si possa dire quando siano stati costruiti, il claustrum (1197), un palatium (1198), il capitolo (1199), una domus provvista di un portico (1212) e di un camino (1218). Affacciata su un brolo (1218) è la casela qua est in pomerio eiusdem ecclesiae (1214), probabilmente la stessa che in altro documento è la domucula brolii (1234), una volta (ambiente voltato) (1235) e un porticus broleti (1299) 49 .

${ }^{36}$ G.P. BROGIOLO, Insediamenti, chiese e porti lungo il Basso Adige tra VI e X secolo, Hortus Artium Medievalium, 22, 2016, pp. 417-430, a pp. 427-428.

37 B. LANFRANCHI STRINA, Un documento inedito del 978 su S. Fidenzio di Megliadino, "Boll. Civici Musei di Padova", 52, 1973 pp. 139-141-

${ }^{38}$ M. VIGATO, Il monastero di S. Maria delle Carceri..., p. 19.

39 L.A. MURATORI, Delle antichità estensi ed italiane, I, Modena 1717, p. 281.

${ }^{40}$ Nella copia pervenuta al Muratori la data era del 1107, ma lui stesso aveva segnalato la non coincidenza tra indizione e data. Quella corretta si trova nella trascrizione di un codice trecentesco, segnalata da M. VIGATO, Il monastero di S. Maria delle Carceri..., p. 19.

${ }^{41}$ L.A. MURATORI, Delle antichità estensi..., pp. 284-285; CDP, II, n. 92.

${ }^{42}$ L.A. MURATORI, Delle antichità estensi..., p. 282; CDP, II, n. 34.

${ }_{43}$ Lo ricorda il vescovo Gerardo nel 1181 (L.A. MURATORI, Antiquitates Italicae Medii Aevii, 1-6, Milano 1739-1742, V, col. 263).

${ }^{44}$ CDP, II, n. 126

${ }^{45}$ Rispettivamente DD Friderici I. Diplomata inde ab a. MCLXVIII ad a. MCLXXX, n. 701; KEHR, Italia Pontificia, VII, I, p. 207, sulla scia di un privilegio di Eugenio III del 1145: KEHR, Italia Pontificia, VII, I, p. 206.

${ }^{46}$ Sulle quali vedi VIGATO 1997 e regesti in MALVESTIO 2004, nn. 1, 3, 5, 6, 7, 9, 11, 13, 14, 17, 18, 19, 20, 21, 23, 25, relativi alle donazioni dal 1117 al 1174 .

47 L.A. MURATORI, Delle antichità estensi..., pp. 356-357.

${ }^{48}$ E. MALVESTIO, S. Maria delle Carceri presso Este. Ricerche su una canonica regolare con edizione o regesto di 154 documenti d'archivio (sec. XII-XIV), tesi di Laurea Università di Padova, 2004, p. 124.

${ }^{49} \mathrm{Nel} \mathrm{1165,} \mathrm{marzo} \mathrm{13,} \mathrm{il} \mathrm{testamento} \mathrm{di} \mathrm{Beatrice,} \mathrm{nobildonna} \mathrm{madre} \mathrm{di} \mathrm{Donella,} \mathrm{viene} \mathrm{redatto} \mathrm{in} \mathrm{hospitali} \mathrm{Sanctae} \mathrm{Mariae} \mathrm{de} \mathrm{Carcere} \mathrm{(L.A.} \mathrm{MURATORI,} \mathrm{Delle}$ antichità estensi..., I, p. 324; CDP, II, n. 871). Un altro del 1174, in choro (L.A. MURATORI, Delle antichità estensi, I, pp. 372-373; "Inventario", c. 197r). Nel 1197, giugno 15, in claustro (L.A. Muratori, Antiquitates Italicae..., IV, coll. 61-62); nel 1198, aprile 28, in palatio (L.A. MURATORI, Antiquitates Italicae..., III, coll. 215-216); nel 1199, lug. 12, in capitulo (L.A. MURATORI, Antiquitates Italicae, III, coll 161-162); nel 1212, sett. 9, sub porticu domus (E. MALVESTIO, S. Maria delle Carceri..., n. 65); nel 1214, giu. 4 in la casela qua est in pomerio eiusdem ecclesiae (E. MALVESTIO, S. Maria delle Carceri..., n. 71); nel 1218, feb. 28 in domo ecclesiae in qua est caminus (E. MALVESTIO, S. Maria delle Carceri..., n. 74); nel 1218, mag. 26 in broilo qui est ante domum (E. MALVESTIO, S. Maria delle Carceri..., n. 75); 1233, giu. 16 sub porticali ecclesie (E. MALVESTIO, S. Maria delle Carceri..., n. 102); nel 1234, apr. 25 sub domucula broili (E. MALVESTIO, S. Maria delle Carceri..., n. 106); 1235, feb. 24 sub volta (E. MALVESTIO, S. Maria delle Carceri..., n. 108).

Nel 1299, dic. 13, per la prima volta si cita il monasterium (E. MALVESTIO, S. Maria delle Carceri..., n. 138), che compare poi in tutti gli atti successivi, talora con ulteriori indicazioni: 1299, dic. 14 sub porticu broleti (E. MALVESTIO, S. Maria delle Carceri..., n. 141); 1307 , gen. 12 in curia monasterii (E. MALVESTIO, S. Maria delle Carceri..., n. 145). 


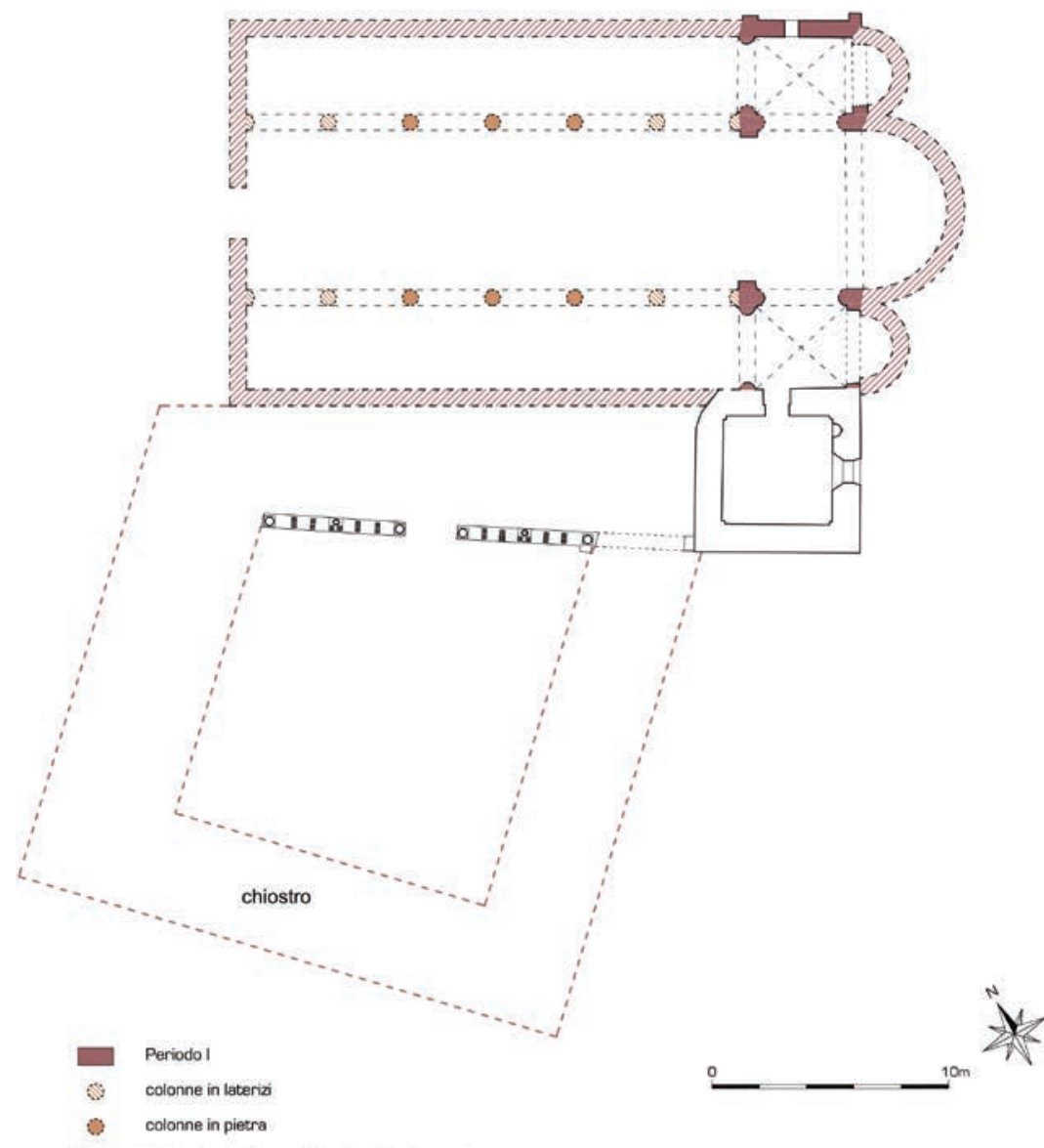

Fig. 15. Carceri, Santa Maria, le strutture conservate e, a tratteggio, la ricostruzione della pianta sulla base delle misure rilevate, nel 1489, nella visita pastorale del vescovo Barozzi.

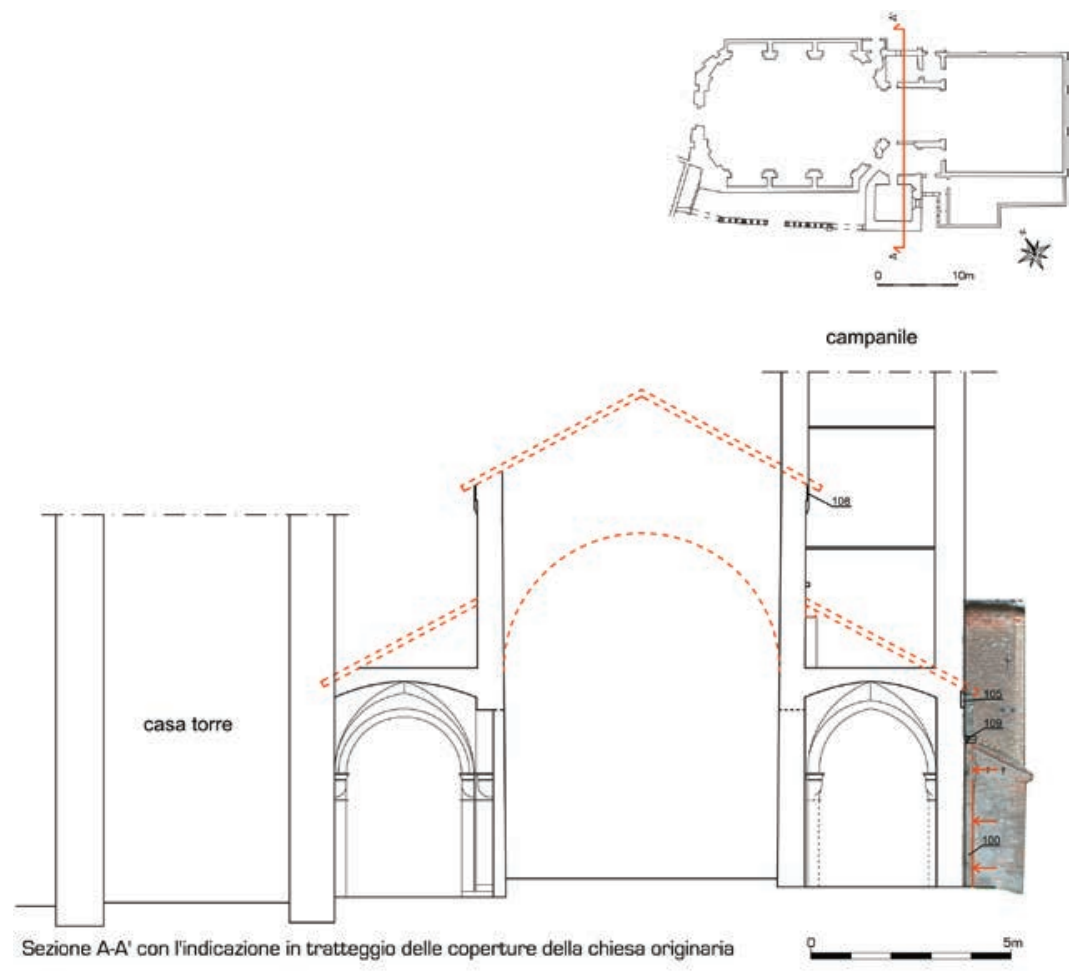

Fig. 16. Carceri, Santa Maria, sezione della chiesa (ricostruita in base a quanto si conserva in elevato) e della casa torre.
Nel $1242^{50}$ un incendio investe la chiesa e il campanile (per il quale fornisce un termine ante quem). Se ne vedono le tracce sulle murature medievali superstite nella navatella nord, annerite del fumo, ma non conosciamo l'entità dei danni e dei conseguenti restauri. Nelle forme attuali, la chiesa è il risultato di ulteriori trasformazioni: nel 1350 viene aggiunto un altare con molte reliquie e nel 1399 la chiesa è nuovamente riconsacrata, plausibilmente dopo una più corposa ristrutturazione, che potrebbe aver compreso la creazione del coro ad oriente dell'abside centrale ${ }^{51}$. Dopo un ulteriore incendio nel 1643, la chiesa venne ricostruita, entro il 1686; nelle forme attuali ad un'unica navata. Infine, dopo la soppressione del monastero, si susseguirono successive fasi di demolizione del chiostro più antico e di altri edifici.

Nonostante questi radicali interventi, per salvare il campanile, impostato sull'ultima campata della navatella nord, e l'antica domus, addossata alla campata sud, è stato conservato, escluse le absidi, l'intero settore orientale della chiesa (fig. 15-16), ovvero parte: (1) del perimetrale nord, con due fasi costruttive anteriori al campanile ${ }^{52}$ (fig. 17) che permettono di ricostruire l'andamento della falda del tetto, visibile sul lato est del campanile (fig. 18), (2) dei due divisori tra navate. La faccia nord del muro settentrionale (visibile all'interno del campanile), dopo una fila di mattoni sporgenti della parete, termina in alto con un motivo decorativo in laterizi,, composto da archetti con peduccio, fregio a dente di sega, corso di mattoni, fregio a rombi, altri due corsi di mattoni (fig. 19), (3) del perimetrale sud, pur se interamente rivestito da intonaci più recenti, (4) delle volte sostenute da semicolonne delle due campate superstiti (fig. 20).

Quanto si conserva della chiesa romanica trova corrispondenza negli atti della Visita del vescovo Barozzi del 1489: la chiesa era a tre navate, quella centrale larga m 6,95 (4 passi), le laterali 3,45 (2 passi), per una lunghezza complessiva di $\mathrm{m} 20,85$ (12 passi),

Le navate erano divise, per ciascun lato, da sei arcate sostenute da: due colonne intere in laterizio, tre di marmo al centro, due semicolonne nella controfacciata e nel muro di testata presso l'abside. Delle tre absidi semicircolari, quella centrale, larga quanto la navata mediana, aveva tre finestre, le laterali solo una. Le pareti della navata centrale erano alte quanto quella meridionale (in quanto a questa si addossava il chiostro). Cinque erano le finestre nel perimetrale sud della chiesa, due in quello nord; in facciata, al di sopra della porta, vi era una bifora, una finestra in corrispondenza delle navate laterali.

Lungo la parete nord (ab ea autem parte quae ad septentrionem vergit, ultra extremam deambulationem) vi erano tre absidi con altrettanti altari, quella centrale alta $\mathrm{m} 1,78$ ( 5 piedi), le laterali 2,86 (8 piedi). Infine sono ricordati un pulpito in marmo sostenuto

50 Necrologium carcerense in G.B. MITTARELLI - A. COSTADONI, Annales camaldulenses, VII, Venetiis 1762, p. 378.

${ }^{51}$ G. ZATTIN, Il monastero di Santa Maria delle Carceri, Padova 1973, p. 79.

${ }^{52}$ Nella faccia interna la muratura ha dapprima paramento in mattoni, rivestiti da intonaco con letti di malta marcati da ampie stilature e poi pietre spaccate in opera incert). La parte bassa è in fase con una semicolonna che sostiene un capitello cubico, in laterizi e la soprastante cornice in pietra a doppia dentellatura, sulla quale si imposta la volta a crociera in laterizi e pietre. 


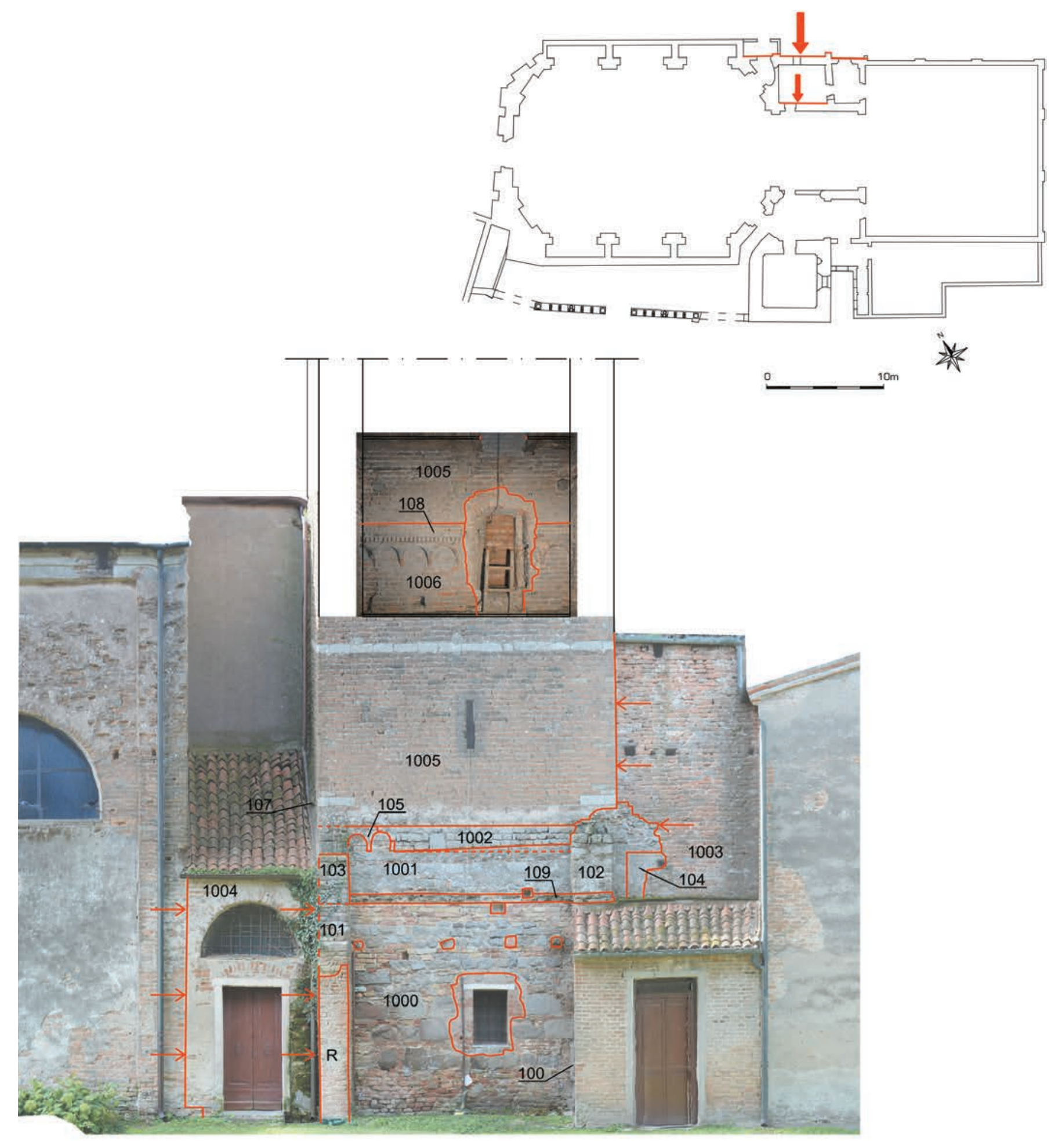

Fotopiano parete nord del campanile e esterno nord della navata centrale

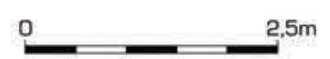

Fig. 17. Carceri, Santa Maria, perimetrale nord della navata centrale, sul quale si imposta il campanile (in alto) e della navata laterale, scandita da due lesene di ineguale dimensione (più larga è quella verso ovest), con due fasi costruttive anteriori al campanile. La prima ha, nella parte bassa, un paramento di pietre lavorate di trachite, di varia dimensione e laterizi romani; seguono poi due fasce irregolari di pietre spaccate e di laterizi terminanti verso l'alto con due corsi aggettanti di mattoni; al di sopra vi è un corso di mattoni e poi tre di pietre spaccate, poi ancora uno di mattoni e uno di pietre spaccate sul quale si impostano almeno cinque corsi di mattoni con una decorazione ad archetti in cotto (due quelli conservati) sormontati da un corso di laterizi. Riferibili ad una seconda fase costruttiva $e$ anteriori alla costruzione del campanile sono tre corsi regolari di bozze che risarciscono la parte alta della muratura, dopo l'eliminazione di quasi tutta la cornice ad archetti, dovuta forse ad una risistemazione della copertura .

da colonnee, al di sotto, un bellissimo fonte battesimale, pur se la chiesa non aveva cura d'anime.
Confronti occidentali abbiamo per i capitelli cubici e le semicolonne in laterizio, attestate a Lomello fin dal Mille e a Nonantola ancora nel 1121, mentre per le cornici in pietra 


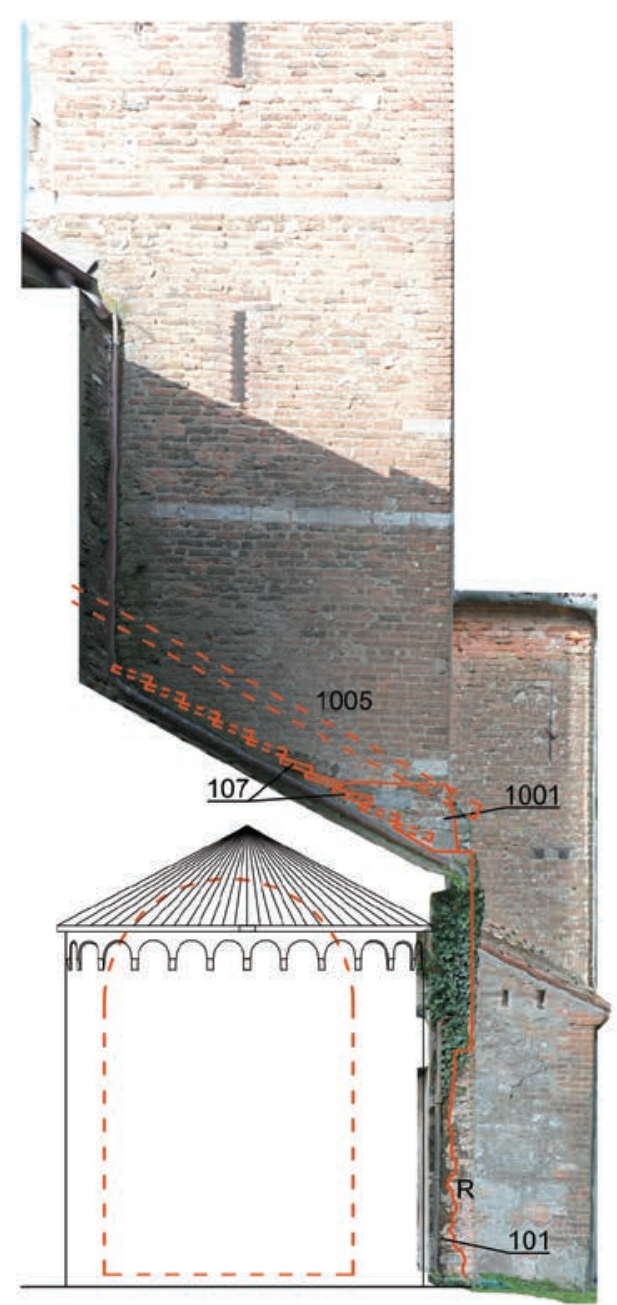

Fotopiano parete est del campanile con la ricostruzione dell'abside piccola a nord

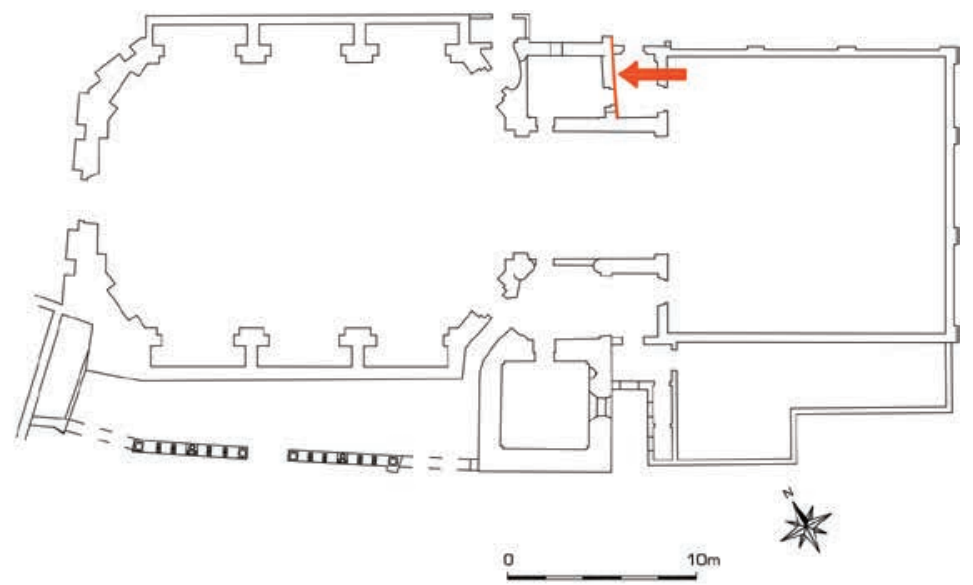

Fig. 18. Carceri, Santa Maria, prospetto est del campanile che si imposta sul muro di testata orientaele della chiesa.

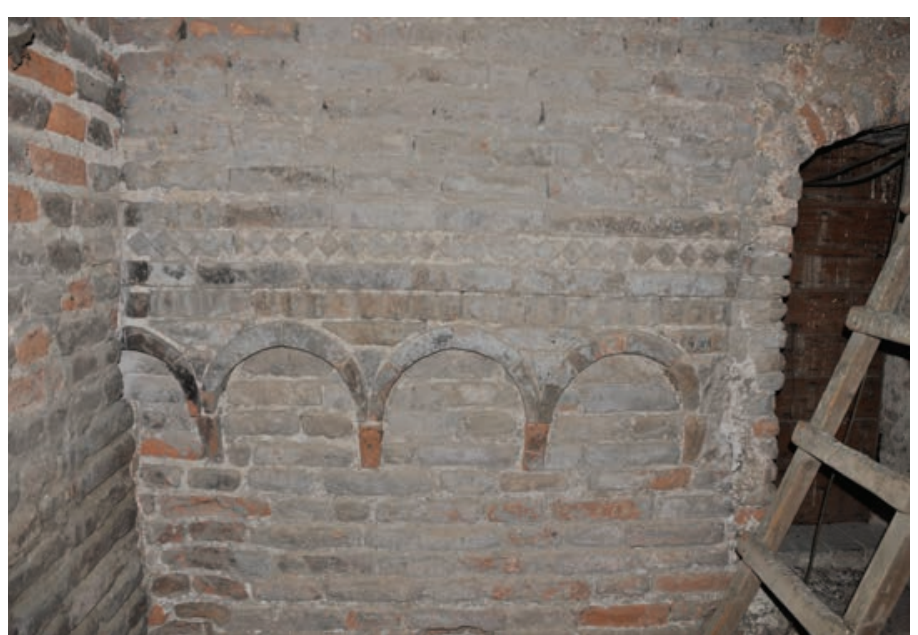

Fig. 19. Carceri, Santa Maria, perimetrale nord della navata centrale (visibile allinterno del campanile), motivo decorativo.

decorate da doppia dentellatura il rapporto più diretto è con le chiese di Santa Sofia e Ognissanti di Padova (datate all'ultimo quarto dell'XI secolo ${ }^{53}$ ), a loro volta con rimandi all'architettura veneziana.

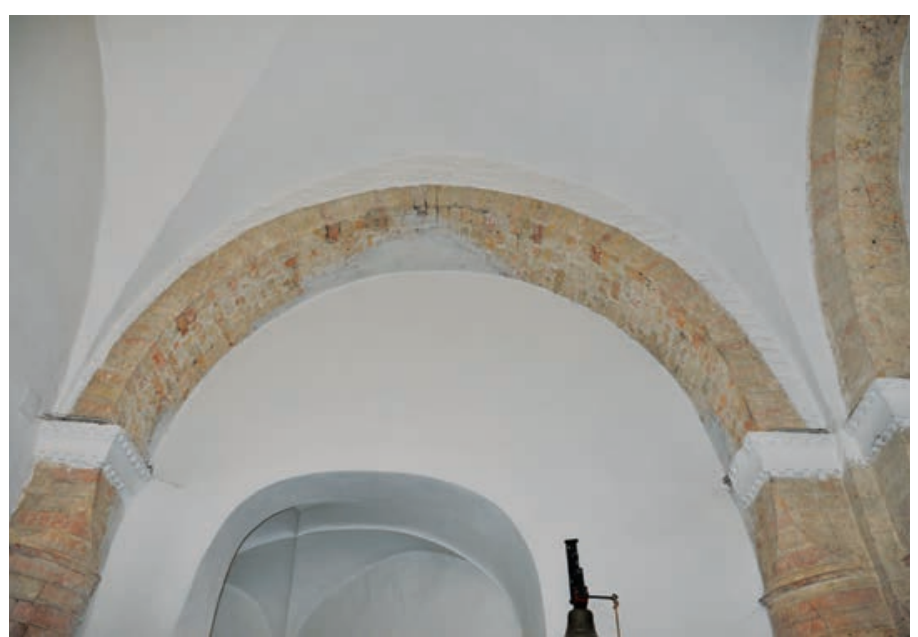

Fig. 20. Carceri, Santa Maria, navatella laterale sud. I pennacchi delle volte a crociera si impostano su semicolonne con collarino, capitelli cubici in laterizio (quello di nord ovest della navata nord ha tracce di decorazione a fresco a linee parallele) e cornici in pietra a gola, decorate da una duplice dentellatura.

Il motivo decorativo composito nel coronamento delle pareti esterne della navata centrale lo si ritrova identico

53 G.P. BROGIOLO, Architetture religiose a Padova alla fine dell'XI secolo, in A. Chavarría Arnau (ed), Padova: architetture medievali, Mantova 2011, pp. 35-74. 

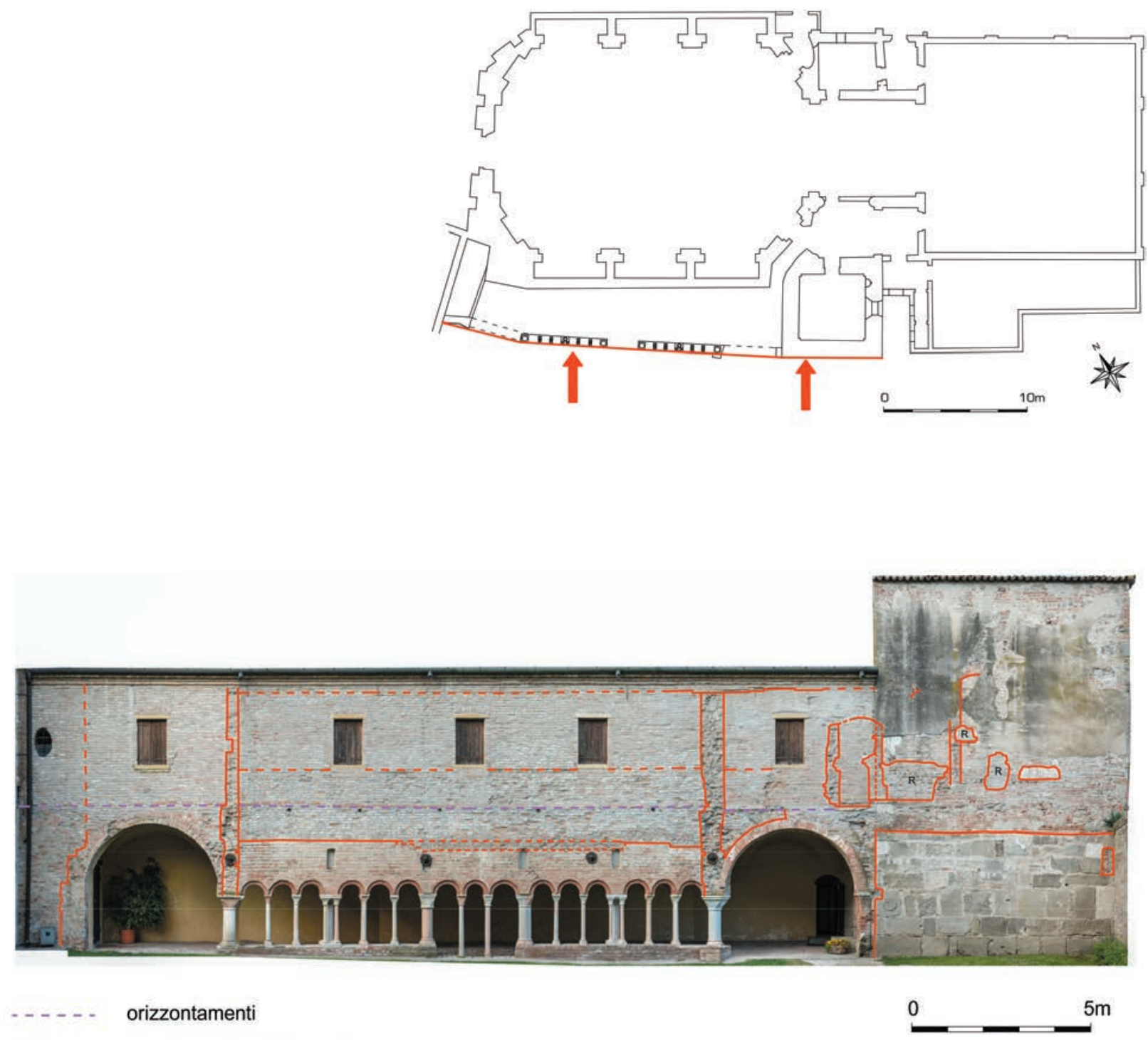

Fig. 21. Carceri, Santa Maria, perimetrale sud della casa torre e portico del chiostro addossato alla chiesa, probabilmente riferibile ai lavori conclusi con la riconsacrazione del 1189.

nell'abside della chiesa di San Salvaro, oggetto della donazione di Alberto Azzo II del 1034, e nel San Martino II di Piove di Sacco, la cui costruzione è stata avviata dal Vescovo Milone nel 1090 e conclusa dal suo successore Pietro nel $1110^{54}$; ha peraltro più generali riferimenti per i singoli elementi che lo compongono, con persistenze fino al XIII secolo ${ }^{55}$.

Nulla osta dunque ad una datazione della chiesa al terzo quarto dell'XI secolo. L'abside centrale, affiancata dalle ultime due campate delle navate laterali, sortiva l'effetto di ampliare il presbiterio, nel quale potevano trovare spazio i presbiteri della collegiata. Accorgimento che si trova in chiese della Lombardia occidentale (come Vimercate Santo Stefano) e del Canton Ticino e a Este nella ricostruzione di Santa Tecla. La pianta deriva da quella romanica di Sant'Ambrogio a Milano, forse terminata da poco, proprio in forme romaniche ${ }^{56}$, quando Alberto Azzo II era conte di quella città.

Anche per la "casa torre", addossata da sud alla chiesa (fig. 21), la domus ricordata nei documenti, i confronti sono con l'architettura padovana della seconda metà dell'XI che, come nel campanile della Vangadizza, riutilizza materiali

${ }_{54}$ G.P. BROGIOLO, La Pieve di San Martino nella Saccisica altomedievale, in Il Duomo di Piove di Sacco, mille anni di storia e arte, Piove di Sacco (PD) 2016, p. 17-52.

55 Contro una cronologia alta della chiesa (attorno al 1078) vi è l'interpretazione di Gian Paolo Trevisan (G. TREVISAN, Santa Maria a Carceri, in F. Zuliani (ed), Veneto romanico, Milano 2008, pp. 265-268) di un "contrafforte a sperone (...) allineato con l'arco trionfale (...) che nasce probabilmente in area veronese, ma è presente anche nell'abbazia della Vangadizza come pure in alcune aree del mantovano prossime all'abbazia di San Benedetto di Polirone (pieve di Coriano, San Floriano di Nuvolato, Sant'Andrea di Ghisione)" Questo peculiare elemento architettonico, secondo lui in appoggio al divisorio con la navata centrale, che compare a Verona nella chiesa di San Lorenzo (fine dell'XI secolo) e si ritrova ancora nei primi decenni del XII secolo, lo porta a riferire i resti romanici alla chiesa consacrata nel 1189 .

In realtà lo sperone, impostato sulla volta della navata nord, è alto solo $\mathrm{m} 1,28$, è collegato ad una vicina mensola e non è saldamente ammorsato al perimetrale, come ci si aspetterebbe da un elemento strutturale. Credo corrisponda alla base di appoggio della travatura dello spiovente della navata nord, che terminava al livello degli archetti e delle lesene esterne. Questa diversa interpretazione induce a concludere (non essendovi interruzione nella muratura) che anche la decorazione della navata centrale appartenga alla prima fase costruttiva.

${ }^{56}$ H.R. SENNHAUSER, Architetture dell'XI-XII secolo a confronto. Osservazioni tra esempi svizzeri e lombardi, in A. Segagni Mlacart, L.C. Schiavi (eds), Architettura dell'XI secolo nell'Italia del Nord. Storiografia e nuove ricerche, (Pavia 2010), Pisa 2012, pp. 41-46 e tav. 4 a p. 331. 
romani di recupero, mettendoli in bella vista. Il campanile ed il chiostro con colonnette binate e alternate a colonne singole agli angoli e in corrispondenza dell'accesso centrale al cortile, sono stratigraficamente posteriori, forse riferibili agli interventi degli anni '70-'8o del XII secolo, conclusi dalla solenne riconsacrazione del 1189 .

\section{CONCLUSIONI}

I contatti lungo l'Adige si erano andati consolidando, nel 952, con l'istituzione, da parte dell'imperatore Ottone I, della Marca di Verona che assicurava un ampio mercato esteso dalle Venezie alla Carinzia e alla Baviera sul quale si fonda il grande sviluppo di Venezia. La ripresa dei commerci lungo l'Adige era il risultato del consolidamento di nuovi percorsi, intrapreso almeno daI IX secolo, sia dai Bizantini (che nell'VIII secolo avevano costruito i castelli di Brondolo e Cavarzere), sia dai Carolingi che avevano affidato agli Almerici, stanziati nella Scodosia, il controllo del percorso del fiume nei comitati di Monselice e Gavello fino al confine con i Bizantini. Sarà Almerico II, l'ultimo discendente di questa importante famiglia legata alll'impero, a fondare, forse già come mausoleo familiare, la collegiata della Vangadizza che Ugo il grande di Toscana trasformerà in monastero, passato nell'XI secolo agli Obertenghi. Alberto Azzo II e i suoi familiari (oltre al figlio della prima moglie Guelfo IV e al nipote, entrambi duchi di Baviera, ma con interessi ad Este, anche Folco e Ugo, figli di secondo letto) finanziano numerosi interventi sui luoghi di culto del distretto di Monselice, in particolare nel settore occidentale da loro controllato. Fondano l'ospedale di Trecontadi e la chiesa di Santa Maria nel castello di Este, elargiscono donazioni alle chiese di San Salvaro (1034), ristrutturano Santa Tecla di Este, sede del vescovo Sinibaldo nel periodo in cui viene cacciato da Padova dal filoimperiale Pietro (ca. 1110-1120).

La loro attenzione, oltre che sul castello di Este, la residenza ufficiale, si focalizza soprattutto sul monastero della Vangadizza, ricostruito come mausoleo della famiglia nella quale vengono sepolti Alberto Azzo II e la prima moglie $\mathrm{Cu}$ nizza, e la collegiata di Carceri, fondata non lontano da Este in un'area di conquista di nuovi spazi agrari. Gli investimenti nelle architetture, dal X secolo almeno vanno infatti di pari passo, in un contesto storico di forte crescita economica con le bonifiche per nuove terre sottratte alle paludi.

Le tecniche murarie impiegate nel castello e nelle chiese della Vangadizza e di Carceri sono le medesime: paramenti in pietre e laterizi di recupero e nucleo a sacco, messi in opera con molta regolarità. Necessarie per la ragguardevole dimensione degli edifici, queste tecniche richiedono maestranze specializzate e abbondanti materiali di riuso, provenienti talora da luoghi distanti, che solo una committenza legata al vertice del potere poteva reperire: nel caso della Vangadizza sarcofagi da Ravenna e da altri centri del Veneto, il magister da Piacenza, pietre da Verona, da dove veniva pure il particolare dei pilastri pentagonali in facciata.

A seconda dei materiali disponibili e in relazione alle dimensioni e alla differenti percentuali di laterizi e pietre lavorate, cambiava il paramento, talora ancheall'interno del medesimo complesso, come a Carceri, dove vi è una netta distinzione tra il perimetrale nord ed i divisori tra navata centrale e laterali. Sempre Carceri le cornici dentellate dei capitelli in pietra a gola arrivavano forse direttamente da Venezia, mentre capitelli cubici in laterizi, colonne e volte a crociera su solidi archi, elementi architettonici rielaborati nell'architettura centropadana dell'XI secolo, tradiscono maestranze occidentali.

Attorno al 1100, ad imprimere un'accelerazione all'evergetismo degli eredi del marchese, sarà la concorrenza tra il ramo locale rappresentato da Folco, fondatore del monastero di Trecontadi, e quello bavarese, facente capo ad Enrico il Nero, cui si deve almeno la ristrutturazione di Santa Tecla di Este, negli anni in cui il vescovo filopale Sinbaldo dovette abbandonare la sede di Padova che l'imperatore aveva affidato ad un presule a lui fedele. 